\title{
The Microstructure of As-Quenched 12Mn Steel
}

\author{
C.C. Kinney ${ }^{1}$, I. Yi $^{2}$, K.R. Pytlewski ${ }^{1}$, A.G. Khachaturyan ${ }^{1}$, N.J. Kim ${ }^{3}$ and J.W. Morris, Jr. ${ }^{1}$ \\ ${ }^{1}$ Department of Materials Science and Engineering, Univ. California, Berkeley, CA 94720, USA \\ ${ }^{2}$ Technical Research Laboratories, POSCO, Gwangyang, 545-711, Korea \\ ${ }^{3}$ Graduate Institute of Ferrous Technology, POSTECH, Pohang, Korea
}

\begin{abstract}
Fe-12Mn steel has the unique and interesting property that, when quenched to $\alpha$ ' martensite from the austenite phase, it forms an ultrafine grained microstructure that has exceptional resistance to cleavage fracture at cryogenic temperatures. The present research was undertaken to complete the characterization of this microstructure and understand why it forms and why it has such exceptional crack resistance. A combination of EBSD and TEM analysis shows that the microstructure is a dislocated lath martensite in which the laths have the Kurdjumov-Sachs relation to the parent austenite. As in other dislocated lath martensites, the prior austenite grains are divided into packets, each of which contains the 6 (of 24) KS variants that mate with the same $\{111\}$ plane. Uniquely, however, the packets are stacks of thin plates that contain all 6 KS variants. The variants within the plate are organized into 3 pair of twinned KS variants that are elongated along their $\{112\}$ twin planes, rotated $120^{\circ}$ from one another, and interwoven to form the 6-variant plate. The ultrafine grains are the laths themselves; twin boundaries between KS variants are known to provide strong barriers to cleavage crack propagation. This unusual microstructure is apparently due to the transformation path; austenite transforms to the hexagonal $\varepsilon$ martensite phase before its ultimate transformation to $\alpha$, and the 6-variant plate is the preferred element to minimize elastic energy in a microstructure created by a dominant $\rightarrow \rightarrow$ ' transformation path.
\end{abstract}

Keywords: Martensite; Lath martensite; Dislocated martensite; 12Mn steel; Microstructure of steel

\section{INTRODUCTION}

Fe-Mn binary alloys with content near $12 \mathrm{Mn}$ have one of the most interesting, and potentially useful microstructures found in martensitic steels [1,2]. When quenched from the FCC $\gamma$-phase, $\mathrm{Fe}-12 \mathrm{Mn}$ alloys transform into an ultra-fine grained martensite of columnar laths that are less 
than $1 \mu \mathrm{m}$ in diameter and $2-3 \mu \mathrm{m}$ in length (Fig. 1a). Moreover, adjacent laths are crystallographically distinct with the consequence that the effective grain size (the "block" size of the martensite) is essentially the same as the lath size. As a consequence, if the alloy is modified to suppress an unfortunate tendency toward intergranular fracture (which can be done, for example, by adding a microalloy addition of B [2]) the ductile-brittle condition in the asquenched condition can be as low as 77K (Fig. 2).

At the time the original research on Fe-12Mn was done [1,2] it was not at all clear why asquenched Fe-12Mn developed this unique microstructure, though it was suspected that the hexagonal, $\varepsilon$ martensite phase plays an important role. In Fe-12Mn the martensite start temperature, $M_{s \varepsilon}$, for the $\gamma \rightarrow \varepsilon$ transformation is slightly above that, $M_{s \alpha}$, for the $\gamma \rightarrow \alpha$ ' transformation $[3,4]$, so there is a partial transformation to the $\varepsilon$ phase before the $\alpha$ ' appears, and there is a significant residual content of $\varepsilon$ phase in the as-quenched alloy (Fig. 1b). It was also not clear precisely why the effective grain size should be so small, since the crystallographic coordination of laths in conventional low-C dislocated martensite leads to effective grain sizes (block sizes) that are much larger than the lath size [5,8-10].

A partial answer to the latter question, the reason for the ultra-fine grain size, was provided by recent research by Yi and Kim [11], who used high-resolution transmission electron microscopy (TEM) and electron back-scatter diffraction (EBSD) to make three important observations: (1) The prior austenite grains in 12Mn steel are subdivided into "packets" as in the more familiar lath martensitic steels, with the laths within the packet having the conventional KurdjumovSachs (KS) relation with the parent austenite. (2) The columnar laths within a packet are organized into thin plates that stack on one another. (3) The adjacent laths within a plate have a strong tendency to be crystallographic twins of one another.

The latter observation explains the ultra-fine effective grain size of $12 \mathrm{Mn}$ steel. KS variants that are crystallographic twins have different "Bain axes" [8,9] and, hence, correspond to different "blocks" of the packet. The adjacent twinned laths act as independent grains in resisting cleavage fracture [8,12]. However, it remained unclear why Fe-12Mn should quench into this structure, which differs so much from that found in the usual low-C lath martensitic steel. The present research was undertaken to complete the characterization of the microstructure and identify the underlying reasons why it appears.

The present paper includes the description and interpretation of the microstructure. The interpretation of the microstructure is based on the possibility of constructing a dislocated, 6- 
variant composite $\alpha$ ' plate with a $\{110\}_{\alpha}$ interface that is an invariant plane with respect to both a $\{111\}_{\gamma}$ and a $\{0001\}_{\varepsilon}$ plane. The theoretical background required to show this is developed in the companion paper [13]. We then discuss how the intrusion of the $\varepsilon$-phase in the martensitic transformation causes this type of plate to form in preference to the stacked, bivariant plates that build the microstructure of low-C lath martensitic steels [10].

\section{EXPERIMENTAL}

The work that is reported here amalgamates two different sets of experiments, one set done at POSTECH by Yi and Kim, and the second done at Berkeley by Kinney, et al.

The Fe-12Mn steel used for the Berkeley work was provided by POSCO as a hot-rolled plate $2.54 \mathrm{~cm}$ in thickness. Its composition was (in wt. \%) $\mathrm{Fe}-0.009 \mathrm{C}-12 \mathrm{Mn}-0.01 \mathrm{Ni}-0.011 \mathrm{~S}-$ $0.023 \mathrm{Cr}-0.02 \mathrm{Nb}$, with less than $0.01 \%$ of Mo, P, and Si. Samples $10 \mathrm{~mm}$ x $10 \mathrm{~mm}$ x $55 \mathrm{~mm}$ were cut from the plate and vacuum encapsulated in quartz tubing prior to any heat treatment. Samples were austenitized at $1473 \mathrm{~K}$ for $1.5 \mathrm{hrs}$, quenched to $448 \mathrm{~K}$, then re-austenitized at $1273 \mathrm{~K}$ for $5 \mathrm{hrs}$ before being quenched to $288 \mathrm{~K}$. The austenitization treatments produced a prior austenite grain size of approximately $100 \mu \mathrm{m}$.

The principal characterization tool used in this research was electron back-scatter diffraction (EBSD). The specimens used for EBSD analysis were approximately $10 \mathrm{~mm} \times 10 \mathrm{~mm} \times 5 \mathrm{~mm}$ pieces, cut from the heat treated bars and encased in metallographic mounts. The samples were mechanically polished with successively finer silicon carbide sheets, then further polished with diamond slurries and an alumina suspension. A final polish with colloidal silica was used to obtain a damage free surface suitable for EBSD examination.

Two EBSD systems were available and used for the analysis, an Oxford Aztec system on a Quanta 3D FEG and a TexSEM Laboratories (TSL) systems in a JEOL SEM. The EBSD scans were performed with a step size of $100 \mathrm{~nm}$, or to detect the epsilon phase, a step size of $60 \mathrm{~nm}$. The data were processed with grain dilation provided by TSL-OIM software, and points with a Confidence Index (CI) of less than 0.02 were omitted. In the inverse pole figure images, a black rotation angle boundary was drawn for any interfacial angle between $0.1-180^{\circ}$. These were included to show the boundaries between crystallographic variants as clearly as possible. The colored EBSD images presented in this study were also created with TSL-OIM analysis software, the tolerance of all colored areas was between $4-4.5$ degrees. The overlays of the 
experimental and theoretical pole figures were created by first rotating the experimental data, again using TSL-OIM software, and then lowering the levels to produce a greyed out pole figure before superimposing the theoretical pole figure.

The TSL-OIM software was also used to determine the thickness of the residual epsilon particles in the final microstructure. The TSL-OIM Analysis software has the ability to quickly isolate a single phase particle present in a rastered scan, measure the dimensions of the particle in the plane of the sample surface (in our case the projected thickness of the plate) and determine the angle between the surface of the sample and the $(0001)_{\varepsilon}$ basal plane of the $\varepsilon$ plate. Given this information the thickness of the plate in the $[0001]_{\varepsilon}$ direction is calculated as described below .

The work done by $\mathrm{Yi}$ and Kim at POSTECH used an Fe-12Mn plate, that was austenitized at $1423 \mathrm{~K}$, rolled 6 times in the recrystallization region to a final thickness of $11.5 \mathrm{~mm}$, and air cooled at $10^{\circ} \mathrm{C} / \mathrm{sec}$. The phase fractions were measured using X-ray diffraction and the Rietveld method [14]. $\mathrm{Cu} \mathrm{K} \mathrm{K}_{\alpha} \mathrm{X}$-rays $(\lambda=1.54056 \AA)$ was used for the X-ray diffraction analysis. For TEM observation, specimens were mechanically polished to $100 \mu \mathrm{m}$ thickness, punched to prepare $3 \mathrm{~mm}$ diameter disks, and then electrolytic polished using a solution of $1000 \mathrm{ml} \mathrm{CH} 3 \mathrm{COOH}+$ $30 \mathrm{ml}$ perchloric acid. The disks were observed using a TEM operating at an acceleration voltage of $200 \mathrm{kV}$. The CaRIne crystallography v3.1 Software was used to determine the crystal orientation relationships from the diffraction patterns. Electron back-scattered diffraction (EBSD) analyses were also used to determine crystal orientations. The EBSD specimens were carefully polished using colloidal silica to reduce the residual stress on the surface area.

\section{ReSUlts}

A prior austenite grain in quenched $\mathrm{Fe}-12 \mathrm{Mn}$ is composed primarily of fresh $\alpha$ ' martensite, with a small admixture of hexagonal $\varepsilon$ phase (about $9 \%$ by volume) and a smaller residue of retained austenite $(\gamma)$. Since the $\alpha$ martensite predominates, we first describe its microstructural pattern, and then discuss the pattern of the fine-scale $\varepsilon$ phase that is interspersed within it.

\subsection{The microstructure of $\alpha^{\prime}$ martensite in as-quenched 12Mn steel}

Fig. 3 is an EBSD map that shows the general microstructure of the $\alpha$ ' phase in $12 \mathrm{Mn}$ steel. The map includes several grains near the brittle fracture surface of a sample broken at $77 \mathrm{~K}$ (the intergranular fracture is so brittle that the grains are not deformed). The figure also includes the 
$(001)$ and $(011)$ pole figures of the prior austenite grain outlined in the figure, showing the orientations of the laths (crystals) it contains. The patterns that appear in these pole figures are typical of all the prior austenite grains examined.

The pole figures shown in Fig. 3 have the usual form for grains whose $\alpha$ ' laths have the Kurjumov-Sachs (KS) crystallographic relation to the parent austenite:

$$
\begin{aligned}
& \{011\} \|\{111\} \\
& \langle 111\rangle \|\langle 110\rangle
\end{aligned}
$$

that is, close-packed planes and close-packed directions are nearly parallel at the $\alpha-\gamma$ interface. This result is confirmed by the comparative data presented in Fig. 4. The diffuse spots in the pole figures show the location and spread of the experimental data; the discrete points give the theoretical location of the poles associated with the $24 \mathrm{KS}$ variants. The theoretical and experimental pole positions superimpose. The data confirm that all $24 \mathrm{KS}$ variants are present in this prior austenite grain, as they are in virtually every prior austenite grain in Fe-12Mn that has been analyzed.

The 24 distinct variants of the KS relation for the FCC-BCC transition follow from the 24 different ways to achieve the orientation relations in eq. (1) [8]: there are 4 independent choices for the $\{111\}$ plane, 3 independent choices for the close-packed $\langle 110\rangle$ direction in each plane, and 2 independent choices for the close-packed $\langle 111\rangle$ direction for a given $\langle 110\rangle$. The six variants that are associated with the (111) plane are illustrated in Fig. 5. The two variants that share a common $\langle 111\rangle$ direction have close packed directions parallel to $[11 \overline{1}]$ (direction 1) and $[\overline{1} 1 \bar{l}]$ (direction 2 ); they are twins of one another, and are marked in the figure by dark and light shades of the same color (red, blue or green). The $3\langle 110\rangle$ directions in the (111) plane locate 3 pair of twins, rotated $120^{\circ}$ from one another about the $[111](=[011])$ axis. A further important feature of a KS variant is its "Bain axis", which identifies the $\langle 001\rangle$ axis of the FCC crystal that is compressed in the "Bain strain" that connects the parent FCC to the BCC cell of the particular variant. There are 2 examples of each Bain variant among the 6 variants in a given $\{111\}$ plane. Note that variants that are twin pairs have different Bain axes.

To capture this information we identify each of the KS variants with a notation of the form aKbc. In this notation a $(=0,1,2,3)$ identifies the $\{111\}$ plane of the KS correspondence for the 
particular variant; $a=1,2,3$ indicates which index in the sequence $\{111\}$ is negative, $a=0$ indicates that all three indices are positive. The letter $K$ shows that the crystallographic correspondence is KS (rather than for example, the Nishiyama-Wasserman (NW) correspondence that is sometimes observed). The index $b(=1,2)$ indicates which of the two possible $\langle 111\rangle$ directions is used, and the index $\mathrm{c}(=\mathrm{X}, \mathrm{Y}, \mathrm{Z})$ identifies the Bain axis.

Having established the KS orientation relation and identified the KS variants, the pattern of the microstructure can be revealed at successive levels of magnification. We first determine the packet structure of the prior austenite grains. In dislocated martensite the KS variants that share a common $\langle 011\rangle$ axis (or, equivalently, have close-packed planes parallel to the same $\{111\}$ plane) are gathered together into packets. The packets can be identified in EBSD maps by using the same color for all laths that have a common $\langle 011\rangle$ axis.

The packet structure in a section through a typical prior austenite grain in as-quenched Fe-12Mn is shown in Fig. 6. Since there are $4\{111\}$ planes, there are 4 distinguishable packets, colored orange, yellow, purple and gray in the figure. This result is typical; virtually all prior austenite grains examined in $12 \mathrm{Mn}$ steel contain all 4 packets, and are expected to for reasons we shall discuss below. The (011) pole figure of this grain is included in the figure, with the poles colored according to their packet. The pole figure contains four rosettes of poles, each centered around a central spot that contains the pole of the common $\langle 011\rangle$ axis of its particular packet along with the tightly clustered poles of all of the laths within the packet.

The location of the $\langle 011\rangle$ packet axes in the pole figure identifies their orientations with respect to the plane of the figure. Fig. 6 shows the projections of the $\langle 011\rangle$ packet axes in the plane of the coss-section and the angles between the packet axes and the normal to the cross-section. The gray packet is tilted by $80^{\circ}$; its (011) plane is almost edge-on in the field of view. The yellow packet is tilted by $5^{\circ}$, so its $(011)$ plane is almost parallel to the field of view.

Appropriately colored EBSD maps show how the various KS variants are distributed within the packet. In particular, the distribution of KS variants within the gray and yellow packets reveals the patterns of the KS variants parallel and perpendicular to the $\langle 011\rangle$ packet axis.

Fig. 7 shows the structure of the gray packet, whose axis is tilted $80^{\circ}$ from $\langle 011\rangle$. Fig. 7 also includes magnified views of two regions of the gray packet. The variants within the packet are colored according to the scheme in Fig. 5; shades of the same color are used to identify twinned variants. As shown in the figure, while there is some irregularity in the pattern, the variants tend 
to be organized into multivariant plates that are approximately $1 \mu \mathrm{m}$ in thickness and, as can be seen from Fig. 6, are stacked perpendicular to $\langle 011\rangle$. Adjacent laths within the plate are ordinarily twins of one another, and these twinned pairs tend to be repeated for some distance along the plate. However, there is an eventual shift to a different twin pair, with the consequence that all 3 twin pairs (all $6 \mathrm{KS}$ variants) are found in each of the plates.

This structure is confirmed by transmission electron microscopy at higher magnification. Fig. 8 shows adjacent laths of dislocated martensite in a plate, and includes diffraction patterns that document the twin orientation across the lath boundary perpendicular to the plane of the plate. Fig. 9 shows parallel plates of dislocated laths, with the laths separated by $\{112\}$ twin planes perpendicular to the plate, and the plates separated by the common (011) plane of the packet.

Fig. 10 includes EBSD maps of the yellow packets in Fig. 6. In this case the packet axis is only tilted $5^{\circ}$ from the normal to the figure, so the map shows the pattern of variants in the $(011)$ plane of the plate. The laths are, again, organized in twin pairs with all three twin pairs present in similar areal fractions. The twin pairs are elongated along the $\{112\}$ twin plane that separates them. Since the three $\{112\}$ twin planes are rotated by $120^{\circ}$ from one another, the three twin pairs are interwoven in the plane of the plate in a triangular pattern. This interweaving also explains why the three twin pairs differ somewhat in shape in perpendicular cross-sections like that shown in Fig. 7; all examples of a particular twin pair have similar orientation in the plane of the plate, and may appear almost square, or as elongated rectangles in a perpendicular cross-section, depending on the orientation of the perpendicular cross-section with respect to the twin pair.

Given the limited number of $\mathrm{KS}$ variants in a given packet, the misorientation angles across the lath boundaries must take one of a few discrete values (Table 1). The angles are readily identified by reference to Fig. 5. Consider a variant that is a member of group 1, such as 0K1X. The other 2 members of group 1, 0K $2 \mathrm{Y}$ and $0 \mathrm{~K} 1 \mathrm{Z}$, are obtained by rotations of $120^{\circ}$. It follows that any interface between two members of group 1 has a misorientation angle of $60^{\circ}$. There are 3 such interfaces. By the same reasoning any one of the 3 interfaces between members of group 2 will also have a misorientation of $60^{\circ}$; there are a total of 6 possible $60^{\circ}$ interfaces. We shall call these rotational boundaries.

Now consider interfaces between members of group 1 and group 2. There are three distinct cases. First, the two variants may be Bain pairs, such as $0 \mathrm{~K} 1 \mathrm{X}$ and $0 \mathrm{~K} 2 \mathrm{X}$. It can be shown that the misorientation angle between Bain pairs is $10.5^{\circ}$. There are 3 interfaces of this kind, which 
we shall call Bain boundaries. Second, the two variants may be twins, such as 0K1X and 0K2Y. Reference to Fig. 5 shows that $0 \mathrm{~K} 2 \mathrm{Y}$ can be obtained from $0 \mathrm{~K} 1 \mathrm{X}$ by rotating $120^{\circ}$ (counterclockwise) to obtain $0 \mathrm{~K} 1 \mathrm{Y}$, then rotating back $10.5^{\circ}$ to obtain $0 \mathrm{~K} 2 \mathrm{Y}$, producing a net misorientation of $70.5^{\circ}$ across the boundary. There are 3 boundaries of this type, which we shall call twin boundaries. The third class includes boundaries between variants in group 1 and 2 that are neither Bain pairs nor twin pairs, for example, that between 0K1X and 0K2Z. From Fig. 5, $0 \mathrm{~K} 2 \mathrm{Z}$ can be obtained from $0 \mathrm{~K} 1 \mathrm{X}$ by rotating clockwise by $120^{\circ}$ to obtain $0 \mathrm{~K} 1 \mathrm{Z}$, then imposing an additional Bain pair rotation of $10.5^{\circ}$ to $0 \mathrm{~K} 2 \mathrm{Z}$. The net misorientation across the boundary is $49.5^{\circ}$. There are 3 boundaries of this type, which we shall call mixed boundaries.

Table 1 lists the various boundary types, and gives their relative frequency, measured as fractions of the total line length in a cross-section, for three cases: a random distribution of KS variants, the microstructure of as-quenched 12Mn steel, and the microstructure of as-quenched 9Ni steel, which has a classic dislocated lath martensite structure [10]. A number of prior austenite grains and orientations were averaged to obtain the data in Table 1.

As expected, the twin boundaries dominate the angle distribution in $12 \mathrm{Mn}$ steel. This is in contrast to the boundary misorientation distribution for conventional dislocated lath martensitic steels, for example, as-quenched 9Ni steel [10] (Table 2) where the predominant boundaries are the low-angle Bain boundaries. This difference reflects the fact that the microstructure of a packet in $12 \mathrm{Mn}$ consists of stacked 6-variant plates that are composed of interwoven twin pairs, while packets in the $9 \mathrm{Ni}$ structure (conventional lath martensite in low-carbon steel) are stacked bivariant plates of Bain pairs [10].

The fraction of rotational $\left(60^{\circ}\right)$ boundaries is $\sim 0.20$ in both cases. However, the role of these boundaries is different in the two microstructures. In $9 \mathrm{Ni}$ steel the bivariant plates that are stacked into a packet are rotated $120^{\circ}$ from one another, providing $60^{\circ}$ boundary misorientations when the variants that meet at the boundary are from the same set (1 or 2) and $49.5^{\circ}$ misorientations (mixed boundaries) when they are from different sets. In $12 \mathrm{Mn}$ steel the twin pairs within the plates are rotated $120^{\circ}$, providing $60^{\circ}$ misorientations when the variants at the boundary between twin pairs have the same class, $49.5^{\circ}$ misorientations when the variants have different class. In $12 \mathrm{Mn}$ steel the fractions of rotational $(0.20)$ and mixed boundaries $(0.17)$ are similar, suggesting that there is, at most, a slight preference for the rotational boundary between twin pairs. However, in $9 \mathrm{Ni}$ steel there is a strong preference for rotational boundaries (0.20) over mixed boundaries (0.03). It is not clear whether this strong preference is due to the relative 
energies of the boundaries or to the mechanistic details of their formation during the martensitic transformation.

It follows that the microstructure of the $\alpha^{\prime}$ phase in as-quenched $12 \mathrm{Mn}$ steel is visually complex, but simple to describe. Each prior austenite grain is subdivided into packets of 4 distinct orientations, corresponding to the $4\{111\}$ planes of the $\gamma$ phase. Each of these packets is a stacking of nominally identical plates that contain the $6 \mathrm{KS}$ variants that have a common $\langle 011\rangle$ axis. The $6 \mathrm{KS}$ variants are divided into 3 twin pairs whose interfaces are the $3\{112\}$ twin planes that are perpendicular to the common $\{011\}$ plane of the packet. The twin pairs are elongated to extend the low-energy twin plane, and are interwoven since the twin planes and, hence, the elongated twin pairs, are rotated by $120^{\circ}$ from one another. The structure of the 6variant plate is shown from the top in Fig. 10, and from the side in Fig. 7. The stacking of 6variant plates to form packets is also illustrated in Fig. 7.

\subsection{The distribution of $\varepsilon$ martensite in as-quenched 12Mn steel}

At a composition of Fe-12Mn the $\mathrm{M}_{\mathrm{s}}$ temperature for the $\rightarrow \quad$ transformation is about $220^{\circ} \mathrm{C}$ while that for the $\rightarrow{ }^{\prime}$ transformation is about $110^{\circ} \mathrm{C}$ [3]. It follows that the transformation of the $\gamma$-phase during quenching initiates with the formation of $\varepsilon$, followed by the formation of $\alpha$, either from the $\varepsilon$ or directly from the $\gamma$. It is, therefore, not surprising to find a significant fraction of retained $\varepsilon$ in the as-quenched microstructure. X-ray analysis of the Postech sample yielded $9 \% \varepsilon, 91 \% \alpha \alpha^{\prime}$. There is also a trace amount of retained $\gamma$, but this was not quantitatively measured.

The crystallography of the retained $\varepsilon$ phase is documented in the transmission electron micrographs presented in Figs. 1 and 11. The bright-field micrograph (Fig. 11(a)) is an edge-on view of the stacked $(011)$ plates in a packet. The dark-field micrograph (Fig. 11(b)) is the image of the $(01 \overline{1} 3)$ spot in the diffraction pattern (Fig. 11(c)), and shows the $\varepsilon$ phase as a mixture of discrete particles and thin films along the plate boundaries. Crystallographic analysis of the diffraction pattern shows that the $\varepsilon$ - $\alpha$ ' orientation relations are

$$
\begin{aligned}
& (0001) \|(011) \\
& {[1 \overline{120}] \|[\overline{11}]}
\end{aligned}
$$


that is, close-packed planes and close-packed directions are parallel, as in the KS relation between $\alpha$ ' and $\gamma$. Since the basal plane of the hexagonal $\varepsilon$ phase, $(0001)$, is congruent with the close-packed plane of the fcc $\gamma$ phase, $(111)$, it follows that the KS orientation relations are automatically satisfied between the $\alpha^{\prime}$ and $\gamma$ phases when eq. (2) holds:

$$
(0001)\|(011)\|(111)
$$

The same crystallographic information is contained in the EBSD maps. Fig. 12 shows a prior austenite grain in which we have identified the packets of $\alpha^{\prime}$ and the islands of $\varepsilon$ phase. There is also a minor fraction of retained $\gamma$, in small, dispersed patches that we did not attempt to identify in the figure. The relations between the 3 phases are apparent in the pole figures shown at right. Given the crystallographic relations between the three phases, the $(111),(0001)$ and $(011)$ pole figures superimpose. The minor fraction of $\gamma$ phase generates 4 poles in the $(111)$ pole figure, showing the orientation of the 4 close-packed $\{111\}$ planes in the parent FCC crystal. The $(0001)$ pole figure has 4 clear spots that superimpose on those in the (111) pole figure. These identify 4 variants of the $\varepsilon$ phase, each of which has its basal plane parallel to one of the 4 close-packed $\{111\}$ planes. The islands that contribute to these poles are colored green, red, magenta and blue in the EBSD map of the grain. The (011) pole figure exhibits the 24 poles of the KS variants gathered, as before, into four rosettes of poles centered about the $\langle 011\rangle$ axes of the $4 \alpha$ packets. As before, the packets are colored orange, gray, purple and yellow in the EBSD map. Their $(011)$ packet axes are tilted from the normal to the plane of the figure by $35^{\circ}$ (orange packet), $40^{\circ}$ (gray), $75^{\circ}$ (purple) and $80^{\circ}$ (yellow). Superimposing the pole figures of the $\alpha^{\prime}$ and $\varepsilon$ phases shows that the $(011)$ plane of each of the $\alpha^{\prime}$ packets lies parallel to the basal plane of the corresponding variant of the $\varepsilon$ phase, in agreement with the first of the orientation relations given in eq. (2). The tilt angles given for the packets also apply to the four variants of the $\varepsilon$ phase and, ultimately, to the four $\{111\}$ planes, in accordance with eq. (3).

The correspondences that appear in the pole figures suggest that there is a unique variant of the $\varepsilon$ phase for each $\alpha$ ' packet, the one variant that satisfies eq. 3. This appears to be the case for the EBSD map shown in Fig. 12, and is more clear in the higher magnification section shown in Fig. 13. This figure shows the green $\varepsilon$ variant decorating the orange packet and the red variant decorating the gray packet, in agreement with the correspondences in Fig. 12. Both sets of $\varepsilon$ islands lie predominantly along $\alpha$ ' plate boundaries, but form separated islands rather than continuous films. There are isolated patches of the blue $\varepsilon$ variant in the purple packet, and only a couple of isolated islands of magenta $\varepsilon$ variant in the yellow packet. The apparent reason for the 
low density of $\varepsilon$ variants in the yellow and purple packets is that their packet axes are almost perpendicular to the plane of the figure. The $\alpha^{\prime}$ plates are viewed edge-on, and many fewer $\varepsilon$ particles are cut than in the shallow-angle slices through the gray and orange packets.

Fig. 13(c) is a magnified view focused on the purple packet and showing the shape and distribution of the $\varepsilon$ phase within it and its immediate surroundings. The orientation of the purple packet is such that the cross-section shown is almost normal to the 6-variant plate, as it is in the adjacent yellow packets. There is relatively little $\varepsilon$ phase visible in purple and yellow packets, but that which does appear has the single variant that satisfies eq. (3). The islands of $\varepsilon$ phase in the purple and yellow packets have roughly equiaxed cross-sections. This is further evidence that the $\varepsilon$ phase exists as discrete islands that are equiaxed particles or short rods, which appear extended in the angled cross-section that appears in Fig. 12 and 13(a).

The only places in the microstructure where the association between the packet and the $\varepsilon$ variant is unclear are the small regions where several packets appear to be intertwined on a fine scale, as in the multicolored region that is embedded in the yellow packet to the left of Fig. 13(a).

These observations are typical of those made on many prior austenite grains. There is a unique association between the orientation of the $\alpha^{\prime}$ packet and the $\varepsilon$ variant that appears within it. The $\varepsilon$ variant is the one that matches its basal plane to the common close-packed plane of the packet, satisfying the orientation relations given in eq. (3) and shown in superimposed pole figures in Fig 13(b).

Fig. 14 illustrates the distribution of $\varepsilon$ phase in a view of the gray packet (Fig. 13(a)) that shows the individual KS variants. The $\varepsilon$ phase is colored yellow for ease of identification, and the KS variants are colored according to Fig. 5. The appearance of the KS variants in the plate is distorted by the high angle of the cut through the plates. Nonetheless the figure does reveal the generally planar configuration of the separate $\varepsilon$ phase particles, which lie primarily along the interfaces between 6-variant plates.

The roughly equiaxed $\varepsilon$ particles that appear in the purple packet, that is, in the cross-section that is perpendicular to the 6 -variant plates, have an average thickness of about $0.08 \mu \mathrm{m}$. The $\varepsilon$ particles that appear in the orange and gray packets are elongated by the angle of the crosssection, which are tilted $35^{\circ}$ and $40^{\circ}$, respectively, from the perpendicular to the plate, and many are extended by thin films that are not seen in the gray and yellow packets. Since the apparent thickness $\left(d^{\prime}\right)$ of a planar film of thickness $(d)$ increases with the angle of the cross-section as 


$$
d^{\prime}=\frac{d}{\sin ()}
$$

where $\theta$ is the angle between the normal to the cross-section and the normal to the film, films whose thickness is below the resolution of the specified step size of the EBSD scan will be invisible in perpendicular cross-sections, but will be visible in angled cuts.

\section{ANAlysis AND Discussion}

\subsection{The microstructural pattern of $\alpha^{\prime}$}

Our results show that the microstructure of as-quenched 12Mn steel, while visually complex is actually straightforward and simple to describe. The pattern is illustrated in Fig. 15. The basic element is a thin, 6-variant plate with faces parallel to $(011)$ that is made up of interwoven twin pairs. Essentially identical plates are stacked to form a packet, and 4 such packets fill the prior austenite grain.

We now face the issue of why this particular pattern is adopted. The combination of experimental and theoretical results [13] supports the following interpretation.

The microstructure that develops during the martensitic transformation of steel is largely determined by the need to minimize elastic energy $[15,16]$, a particularly difficult problem given the severe crystallographic distortion involved in the $\rightarrow$ ' transformation and the irregular shapes of the grains of the polygranular parent austenite. Ideally, the basic element of the transformation is a thin plate that has an invariant plane with the parent phase, in which case its elastic energy is small. If such plates can be joined together without gaps or distortions so that they ultimately utilize all of the KS variants in each prior austenite grain, then the net transformation strain is a simple dilation (expansion or contraction) and a polygranular body of arbitrary geometry can transform martensitically without introducing long-range strains.

We have recently discussed how this is accomplished in conventional, low-C dislocated martensite [16]. If the Bain strain that accomplishes the $\rightarrow$ ' transformation is supplemented by shear by an appropriate set of dislocations, the transformation can proceed through the 
formation of thin, bivariant plates that include the two KS variants that share the same Bain axis, and which fit perfectly on the close-packed planes of the austenite (i.e., have an invariant plane, $\{011\} \|\{111\})$. There are 3 such plates ("blocks") for each packet. The blocks stack along the $\langle 011\rangle$ axis to create a packet whose net shape change is a simple elongation along that axis. In summary, there are three distinct blocks in each packet, each of which is a composite of the two KS variants (from the six) that have the same Bain axis, a structure described in detail by us and others [5-7, 10, 14]. When the prior austenite grain is filled with 4 such packets its net shape change is a simple hydrostatic expansion; polygranular austenite can transform to $\alpha$ ' martensite with the same prior austenite grain structure with no long-range strain.

The microstructure of $12 \mathrm{Mn}$ steel is similar in that its packets are made up of stacked plates that mate on (011) planes and produce packets whose strain is a simple elongation along the packet axis. However, the nature of the plates is quite different. In conventional, low-C dislocated martensite the plates are composed of two KS variants (a bivariant plate) that share a Bain axis so there are only $2 \mathrm{KS}$ variants in each plate; but the plates in 12Mn are made up of 3 twinned pairs of KS variants so that all 6 of the KS variants of the packet are included in each plate. The extended surfaces of the plates are $(011)$ planes, so identical plates can stack in the [011] direction to create a packet that fills space. The microstructural similarity to the stacking of bivariant plates in conventional lath martensite suggests that the 6-variant plate in $12 \mathrm{Mn}$ is an alternative solution to the problem of finding a plate made up of KS variants that has an invariant plane (011) \|(111).

This problem is addressed by $\mathrm{Li}$, et al [13] in the companion theoretical paper. Using the Khatchaturyan [16] formulation of the crystallogrpahic theory of martensite this work establishes three relevant results. First, given the Bain strain that governs the $\rightarrow$ ' transformation, it is not possible to construct a plate that consists of a twinned pair of KS variants and has a (011) ||(111) invariant plane. It is not clear whether it is possible to construct such a plate when all three KS twin pairs are included, but even if it were, such a plate would be energetically unfavorable with respect to the bivariant plate because of its relatively high surface energy. It, therefore, appears that the twinned plates observed in $12 \mathrm{Mn}$ steel do not originate from the $\gamma$ phase. Second, if we assume that the $\alpha$ ' martensite originates from the $\varepsilon$ phase, the transformation strain of the $\rightarrow$ ' transformation also does not allow a twinned KS variant plate with a $(011) \|(111)$ invariant plane. Third, however, given an appropriate distribution of dislocations, a 6-variant plate can be constructed that is made up of the 3 twinned pairs of KS variants that belong in the packet, in equal fractions, and has an invariant plane (011) $\|(0001)\|(111)$. A 6-variant plate of the sort we observe experimentally is, therefore, a 
favorable structure to form from the $\varepsilon$ phase at the $\varepsilon-\gamma$ interfaces that exist in a steel that has partially transformed to $\varepsilon$ martensite before the $\rightarrow$ ' transformation initiates. These plates can grow to consume the $\gamma$ phase by the $\rightarrow \rightarrow$ ' transformation path.

These 6-variant twinned plates can then be stacked to make a packet whose net strain is a simple elongation along the $\langle 011\rangle$ axis of the packet, as in Fig. 15b. The 4 crystallographically distinct packets then combine to fill the prior austenite grain (Fig. 15c). The net transformation strain is a simple dilation, with the consequence that polygranular austenite can transform martensitically without long-range shear.

Given a martensitic transformation from a mixed microstructure of $\gamma$ and $\varepsilon$ it is, of course, possible to initiate the transformation from the $\gamma$ phase rather than the $\varepsilon$ phase. This should produce a packet microstructure of stacked bivariant blocks, as is commonly found in low-C martensite [5,10]. Since these bivariant blocks contain Bain pairs with low-energy interfaces, it is interesting that they are not found in $12 \mathrm{Mn}$ steel, which appears to be made up entirely of the 6-variant plates. The reason for this selection is not entirely clear, but there are two factors that should be relevant. First, as discussed in the companion paper [13], the $\rightarrow$ ' transformation may simply be easier to accomplish since there is a better crystallographic match (smaller strain) for $\rightarrow$ ' than for $\rightarrow$ '. Second, while the 12Mn microstructure stacks identical 6-variant plates that individually have zero lateral shear, the typical low-C martensite must stack 3 distinct bivariant plates to cancel the in-plane shear. This seems difficult to accomplish when the parent structure is a fine-scale intermixture of $\gamma$ and $\varepsilon$. If interference by the $\varepsilon$ particles makes it difficult to create the periodic stacking of bivariant plates that is needed to eliminate the in-plane shear, the 6-variant plate of the $12 \mathrm{Mn}$ structure will be preferred.

It is important to recognize that the heavily twinned microstructure of $12 \mathrm{Mn}$ steel is different in kind and character from the "twinned martensite" microstructures found in high-C and high-alloy martensites $[15,17]$. In "twinned martensite" the $\alpha$ ' forms in bivariant twinned plates that lie on high-index invariant planes. The crystallographically different plates of twinned martensite cannot be stacked efficiently to cancel shear strains, with the consequence that these microstructures have complex, irregular distributions of plates with significant fractions of retained $\gamma$ phase and internal stresses that tend to make them brittle.

In the $12 \mathrm{Mn}$ structure, in contrast, the 6-variant twinned plates are heavily dislocated, and must be dislocated to create the invariant plane. The $12 \mathrm{Mn}$ microstructure is a form of dislocated martensite. The plates are stacked to complete the transformation of austenite while relaxing 
internal stresses so that the steel is ductile (at least it is when intergranular fracture is suppressed). Moreover, since the twin boundaries are high-angle boundaries, the individual martensite laths in $12 \mathrm{Mn}$ steel act as independent blocks in resisting cleavage fracture. As a consequence of its microstructure the alloy has an outstanding combination of strength, ductility and toughness at low temperature in the as-quenched condition.

We finally note that isolated patches of the 6-variant plate have been found by Chen, et al [18] in 304 stainless steel deformed at sub-zero temperatures, and incipient plates were reported by Pisarik and Van Aken [19] in as-cast Fe-15Mn-2Si-1.4Al-0.08C-0.017N. It appears that the 6variant plate may be a common product of the $\rightarrow$ ' transformation, as suggested by the analysis in ref. [13].

\subsection{The distribution of retained $\varepsilon$ phase}

The pattern of the retained $\varepsilon$ phase in $12 \mathrm{Mn}$ steel is illustrated in Figs. 12 and 13. The $\varepsilon$ particles are discrete bodies that tend to lie between $\alpha$ ' plates. They appear as equiaxed particles that are sometimes extended as films along the plate boundaries. The equiaxed particles have a mean thickness of $\sim 0.08 \mu \mathrm{m}$. Each packet contains only one variant of $\varepsilon$ martensite, that particular variant whose basal plane lies perpendicular to the $\langle 011\rangle$ axis of the packet.

While we have not been able to observe the pattern of $\varepsilon$ martensite in the austenite prior to the transformation to $\alpha$, it seems reasonable to expect that it resembles that found in alloys with slightly higher Mn content that quench to a mixture of $\varepsilon$ and $\gamma$. Steels with roughly 17Mn have this behavior [4,20]. Micrographs of the two-phase mixture in $17 \mathrm{Mn}$ steels show the prior $\gamma$ densely decorated with a rather uniform mixture of the four $\varepsilon$ variants, with primary $\varepsilon$ plates traversing the grain. This distribution is dramatically different from that of the retained $\varepsilon$ in $12 \mathrm{Mn}$, where the $\varepsilon$ is in small, discrete particles with each $\varepsilon$ variant confined to a single packet.

The most plausible explanation for this pattern is that the $\varepsilon$ phase is largely consumed by the growing $\alpha$, which dissolves those $\varepsilon$ variants that are not in registry with the growing packet and partially dissolves the $\varepsilon$ plates that are properly oriented, breaking them up into small particles. The ultimate division of the grain into four $\alpha^{\prime}$ packets of nearly equal volume is required to minimize elastic energy, and the relative growth of the $\alpha^{\prime}$ packets during the course of the transformation presumably adjusts itself to accomplish this. 


\section{Conclusions}

On quenching, $\mathrm{Fe}-12 \mathrm{Mn}$ steel transforms martensitically to a two-phase mixture that is primarily (>90\%) BCC $\alpha$ ' martensite with an admixture <10\% HCP $\varepsilon$ martensite with a small residue of the parent $\gamma$ phase. The transformation sequence is such that the alloy has partially transformed to $\varepsilon$ before the $\alpha$ ' transformation begins.

As is common in low-C dislocated lath martensitic steels, the $\alpha$ ' forms dislocated laths that have a conventional KS relation to parent austenite. The laths are gathered into 4 crystallographically distinct packets, each of which contains the $6 \mathrm{KS}$ variants that interface with a particular one of the $4\{111\}$ planes of the austenite. Each prior austenite grain is subdivided into packets that include examples of all 4 packet types.

Where the $12 \mathrm{Mn}$ microstructure differs dramatically from that of conventional lath martensite is in the internal structure of the packets. In 12Mn the six KS variants of the packet form 3 twinned pairs which are extended along the twin planes and interwoven to form thin, 6-variant plates bound by their common $(011)$ planes. The plates are then stacked to create the packet.

A companion theoretical analysis [13] suggests a straightforward explanation for this structure. If the transformation is $\rightarrow{ }^{\prime}$ then the dislocated 6-variant plate has an invariant plane $(011)\|(0001)\|(111)$. It follows that the $\alpha$ ' forms and grows easily at the $\varepsilon-\gamma$ interfaces in the partially transformed alloy. The 6 -variant $\alpha^{\prime}$ plates stack on one another to make a packet whose net strain is a hydrostatic expansion plus a linear stain along the axis of the packet. The 4 packets that fill the prior austenite grain can cancel this linear strain so that the net strain is hydrostatic; the microstructural pattern is such that a polygranualr austenite can transform martensitically to $\alpha$ ' with no long-range shear.

The microstructural distribution of the residual $\varepsilon$ phase is also of interest. The $\varepsilon$ phase appears as discrete particles, sometimes extended as films along the plate boundaries. All of the $\varepsilon$ phase in a given packet has the same variant, the variant whose $(0001)$ basal plane parallels the $(011)$ plane of the packet. Since the $\rightarrow$ transformation that precedes the transformation to $\alpha^{\prime}$ is expected to produce a mixture of all $4 \varepsilon$ variants in extended plates, it appears that the $\alpha^{\prime}$ consumes $\varepsilon$ phase as it grows, eliminating the variants that are incompatible with the packet and breaking up the $\varepsilon$ plates that are retained. 
The twinned KS pairs that are the basic elements of the 6-variant plates are different Bain variants of the KS relation. Since the boundaries between different Bain variants resist the propagation of cleavage cracks, this microstructure has an ultrafine grain size with respect to cleavage and, therefore, can have exceptional cryogenic toughness in the as-quenched condition. This attribute is unique among martensitic steels.

\section{Acknowledgements}

The authors are grateful POSCO for providing the alloys used in this research, and for helpful discussions. This work was supported by the US National Science Foundation under Grant DMR1006160.

\section{REFERENCES}

[1] Hwang, S.K., Morris Jr., J.W., The improvement of cryogenic mechanical properties of Fe-12 Mn and Fe-8 Mn alloy steels through thermal/mechanical treatments, Met. Trans. A, 10, (1979) 545-555

[2] Hwang, S.K. and Morris Jr., J.W., The use of a boron addition to prevent intergranular embrittlement in Fe-12Mn, Met. Trans. A, 11, (1980) 1197-1206

[3] M.J. Schanfein, M.J. Yakota, V.F. Zackay, E.R. Parker and J.W. Morris, Jr., ASTM STP 579, 361-77 (1979)

[4] Y. Tomota, M. Strum, J.W. Morris, Jr., Microstructural dependence of Fe-high Mn tensile behavior, Met. Trans. A, 17, (1986) 537-547

[5] S. Morito, H. Tanaka, R. Konishi, T. Furuhara, and T. Maki, T., The morphology and crystallography of lath martensite in Fe-C alloys, Acta Mater., 51, (2003) 1789- 1799

[6] H. Kitahara, R. Ueji, N. Tsuji, Y. Minamino, Crystallographic features of lath martensite in low-carbon steel, Acta Mater., 54 (2006), 1279-1288

[7] S. Morito, X. Huang, T. Furuhara, T. Maki, N. Hansen, The morphology and crystallography of lath martensite in alloy steels, Acta Mater., 54 (2006), 5323 - 5331

[8] Z. Guo, C.S. Lee and J. W. Morris, Jr., On coherent transformations in steel, Acta Mater., 52, (2004) 5511-5518 
[9] J.W. Morris, Jr., On the ductile-brittle transition in lath martensitic steel, ISIJ Int., 51, (2011) $1569-1575$

[10] C.C. Kinney, K. Pytlewski, A.G. Khachaturyan, and J.W. Morris, Jr., The microstructure of lath martensite in quenched 9Ni steel, Acta Mater., 69, (2014) 372-385

[11] I. Yi, MS Thesis, POSTECH, Pohang, Korea, 2011

[12] J.W. Morris, Jr., C. Kinney, K. Pytlewski and Y. Adachi, Microstructure and cleavage in lath martensitic steels, Sci. Tech. Adv. Mater., 14, (2013) 041208

[13] L. Qi, A.G. Khachaturyan and J.W. Morris, Jr. (submitted)

[14] R.A. Young, The Reitveld Method, Oxford University Press, Oxford, 1993

[15] A.G. Khachaturyan, The Theory of Structural Transformations in Solids, J. Wiley, New York 1983: Chapt. 6

[16] L. Qi, A.G. Khachaturyan and J.W. Morris, Jr., The microstructure of dislocated martensitic steel: Theory, Acta Mater., 76, (2014) 23-39

[17] C.M. Wayman, Introduction to the Crystallography of Martensitic Transformations, Macmillan, New York (1964)

[18] M. Chen and N. Tsuji, Kyoto Univ., Private Communication (2015)

[19] S.T. Pisarik and D.C. Van Aken, Crystallographic Orientation of the $\varepsilon \rightarrow \alpha^{\prime}$ Martensitic (Athermal) Transformation in a FeMnAlSi Steel, Met. And Mat. Trans. A, 45, (2014) 3173-78

[20] K.H. Kwon, B.-C. Suh, S.-I. Baik, Y.-W. Kim, J.-K. Choi, N.J. Kim, Deformation behavior of duplex austenite and $\varepsilon$-martensite high-Mn steel, Sci. Tech. Adv. Mat., 14, (2012) 014204 
Table 1: Boundary Frequencies

\begin{tabular}{|l|l|l|l|l|}
\hline Boundary type & Angle & Fraction - Random & Fraction - 12Mn & Fraction - 9Ni \\
\hline Rotational & $60^{\circ}$ & 0.40 & 0.20 & 0.20 \\
\hline Bain & $10.5^{\circ}$ & 0.20 & 0.25 & 0.60 \\
\hline Twin & $70.5^{\circ}$ & 0.20 & 0.38 & 0.17 \\
\hline Mixed & $49.5^{\circ}$ & 0.20 & 0.17 & 0.02 \\
\hline
\end{tabular}



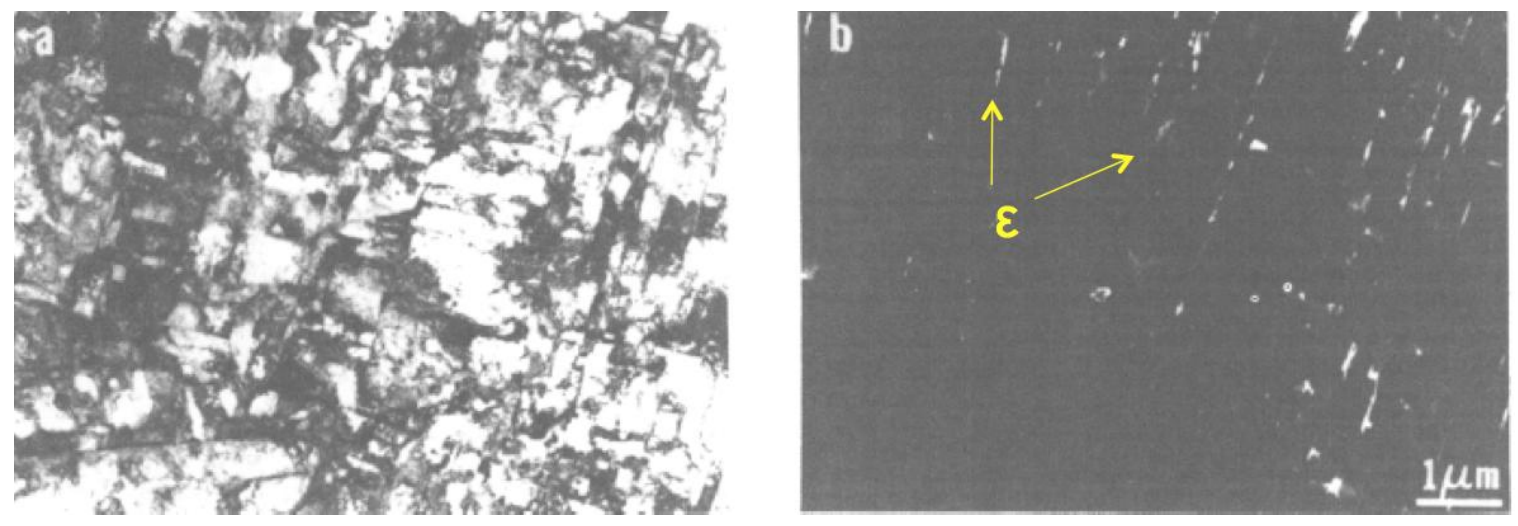

Fig. 1: (a) Bright-field TEM of as-quenched Fe-12Mn-0.1Ti-0.05Al showing fine-grained $\alpha^{\prime}$ martensite. (b) Dark-field TEM showing distributed $\varepsilon$ martensite in the $\alpha^{\prime}$ matrix (from ref. [1]). 


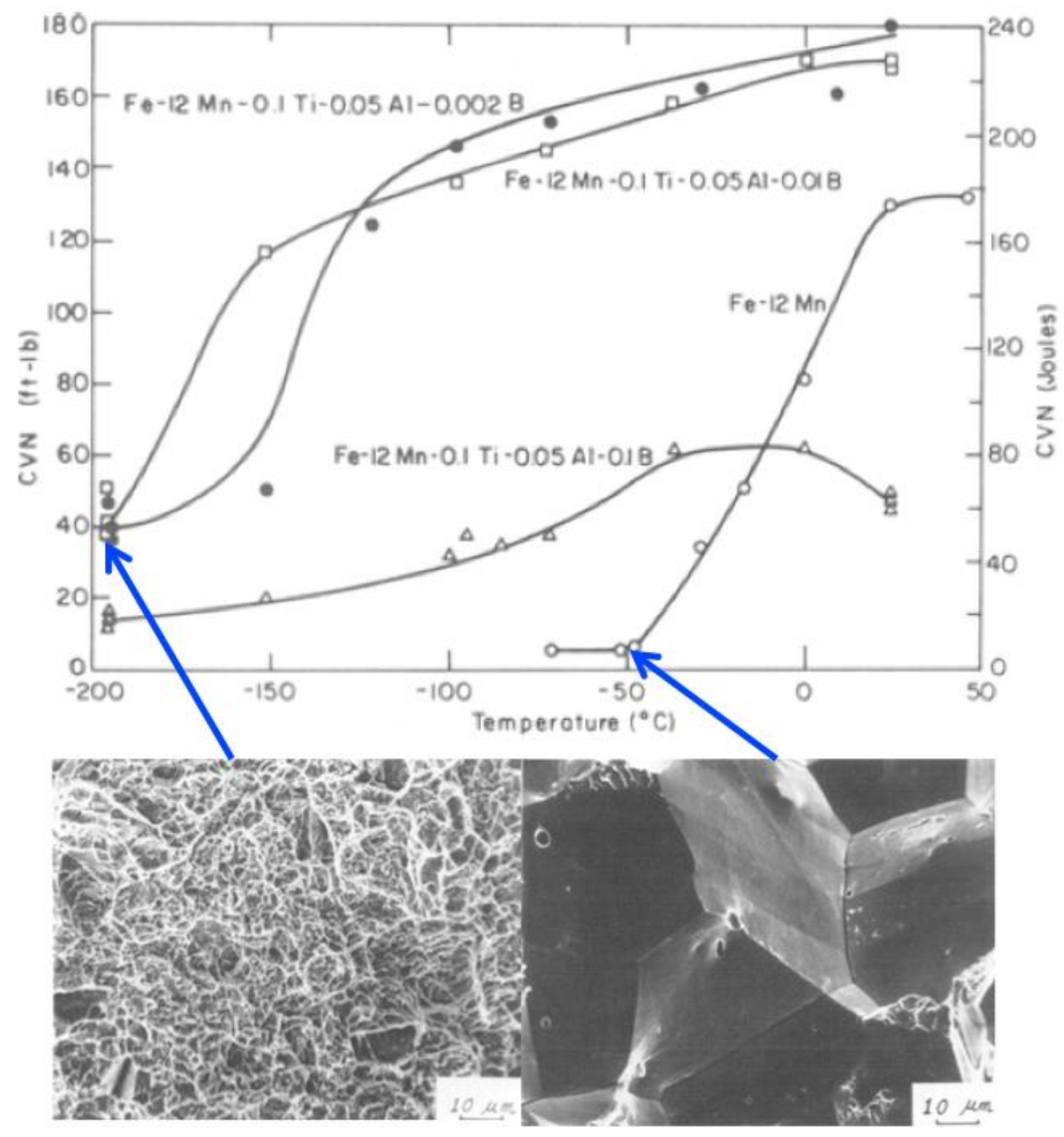

Fig. 2: Adding 0.002B to Fe-12Mn-0.1Ti-0.05 Al changes the low-temperature fracture mode from brittle intergranular to ductile rupture and suppresses the ductile-brittle transition to cryogenic temperatures (from ref. [3]). 

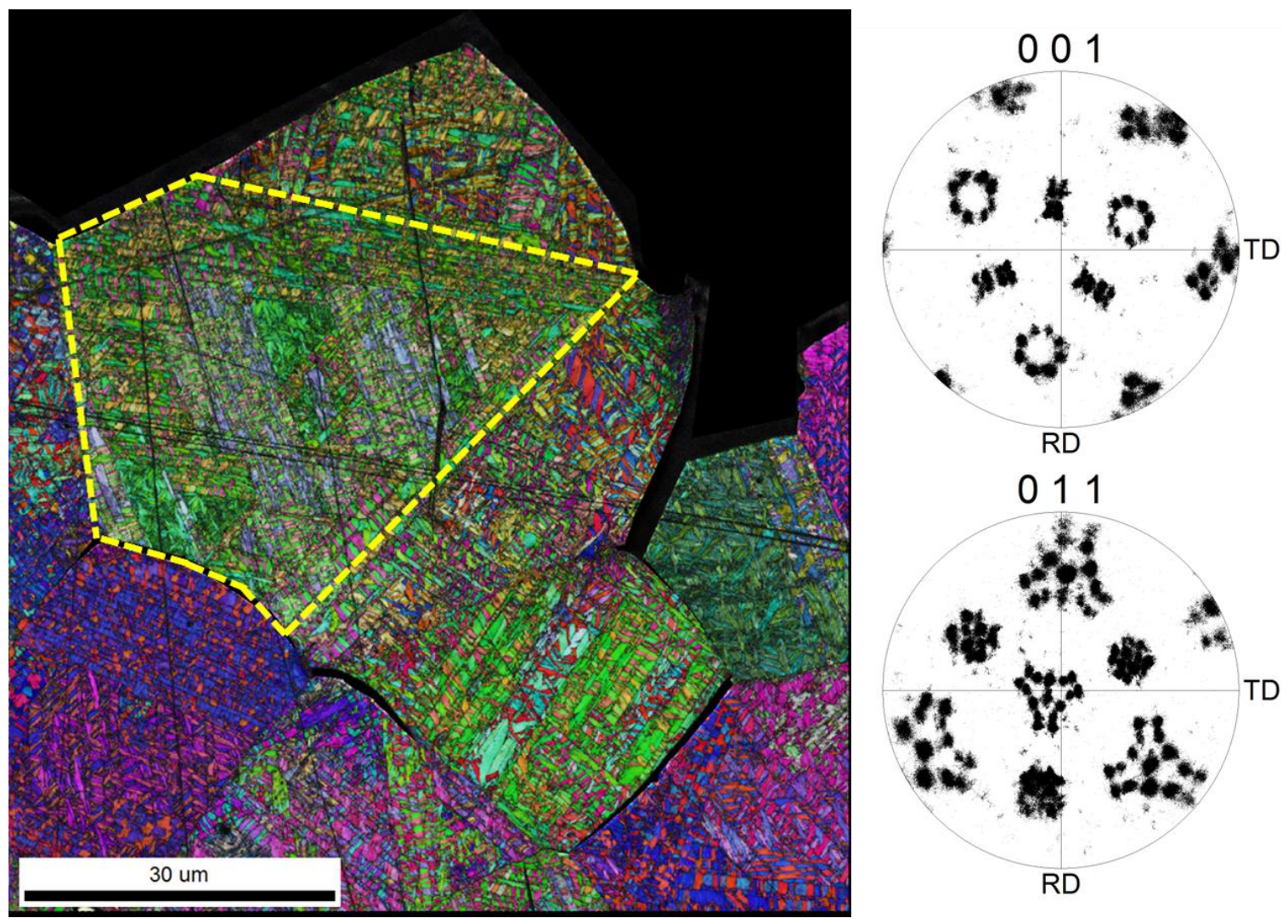

Fig. 3: EBSD map of grains near the fracture surface in $12 \mathrm{Mn}$ steel, showing the $(001)$ and $(011)$ pole figures from the particular grain outlined. 

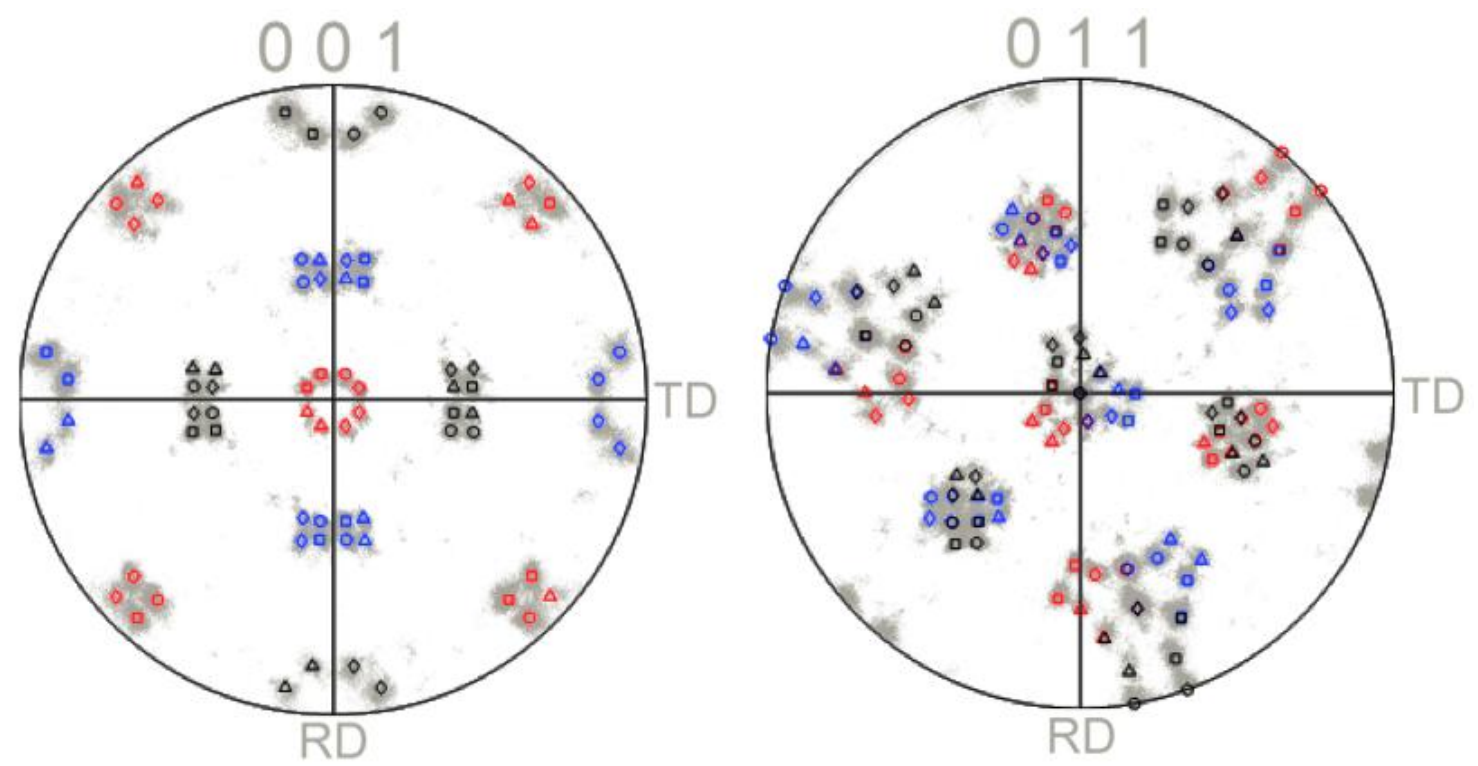

Fig. 4: Pole figures for the prior austenite grain of Fig. 3, replotted to compare the experimental data (diffuse gray spots) with the theoretical patterns for the KS orientation relations (discrete spots). The spots coincide, and all $24 \mathrm{KS}$ variants are present in the grain. The colors of the theoretical spots, red, blue and black, identify the Bain axis of the KS variant associated with the particular spot. 


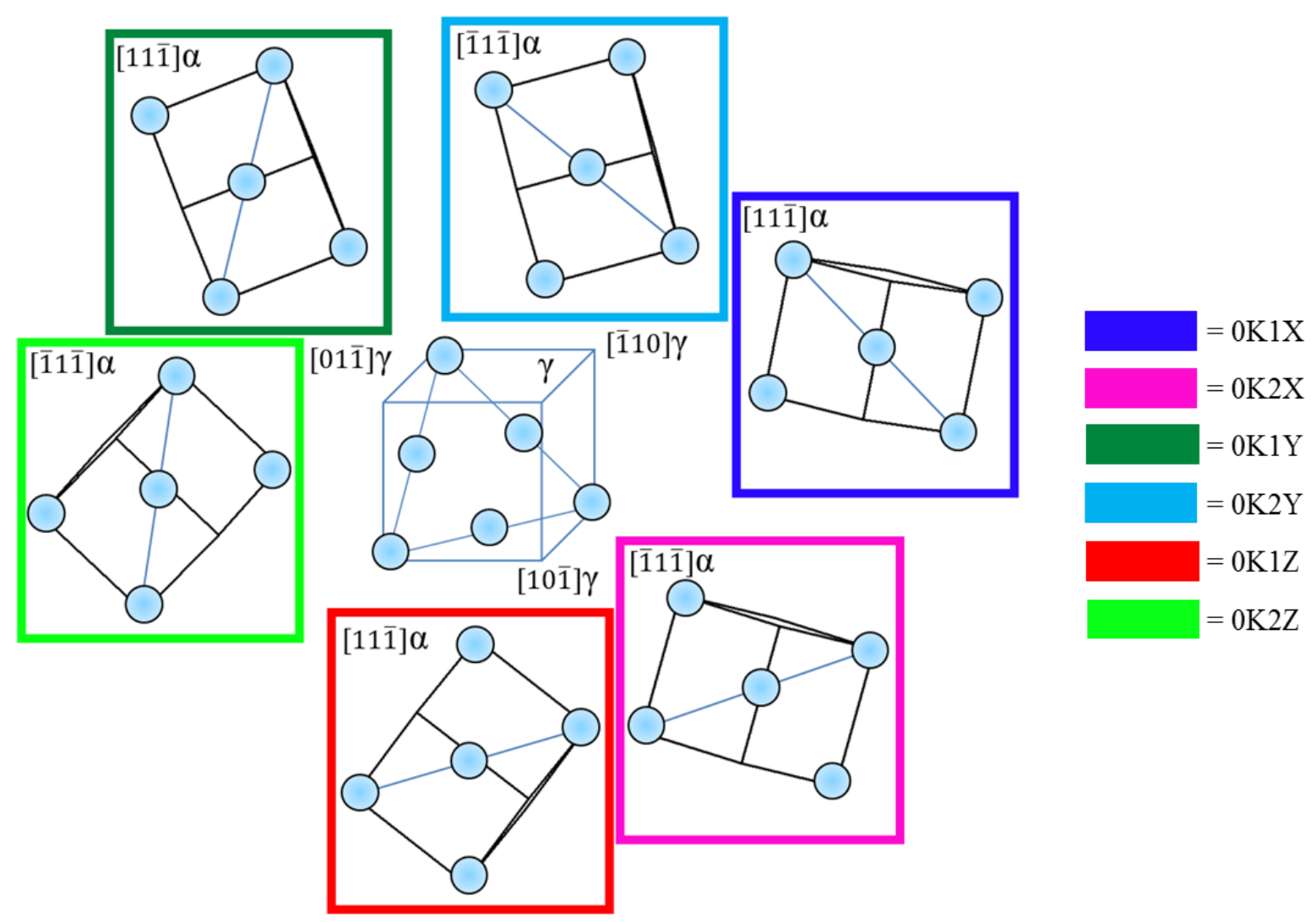

Fig. 5: The $6 \mathrm{KS}$ variants that lie in the (111) plane. The variants are colored so that twinned variants are shades of the same color. The notation that labels the variants is discussed in the text. 


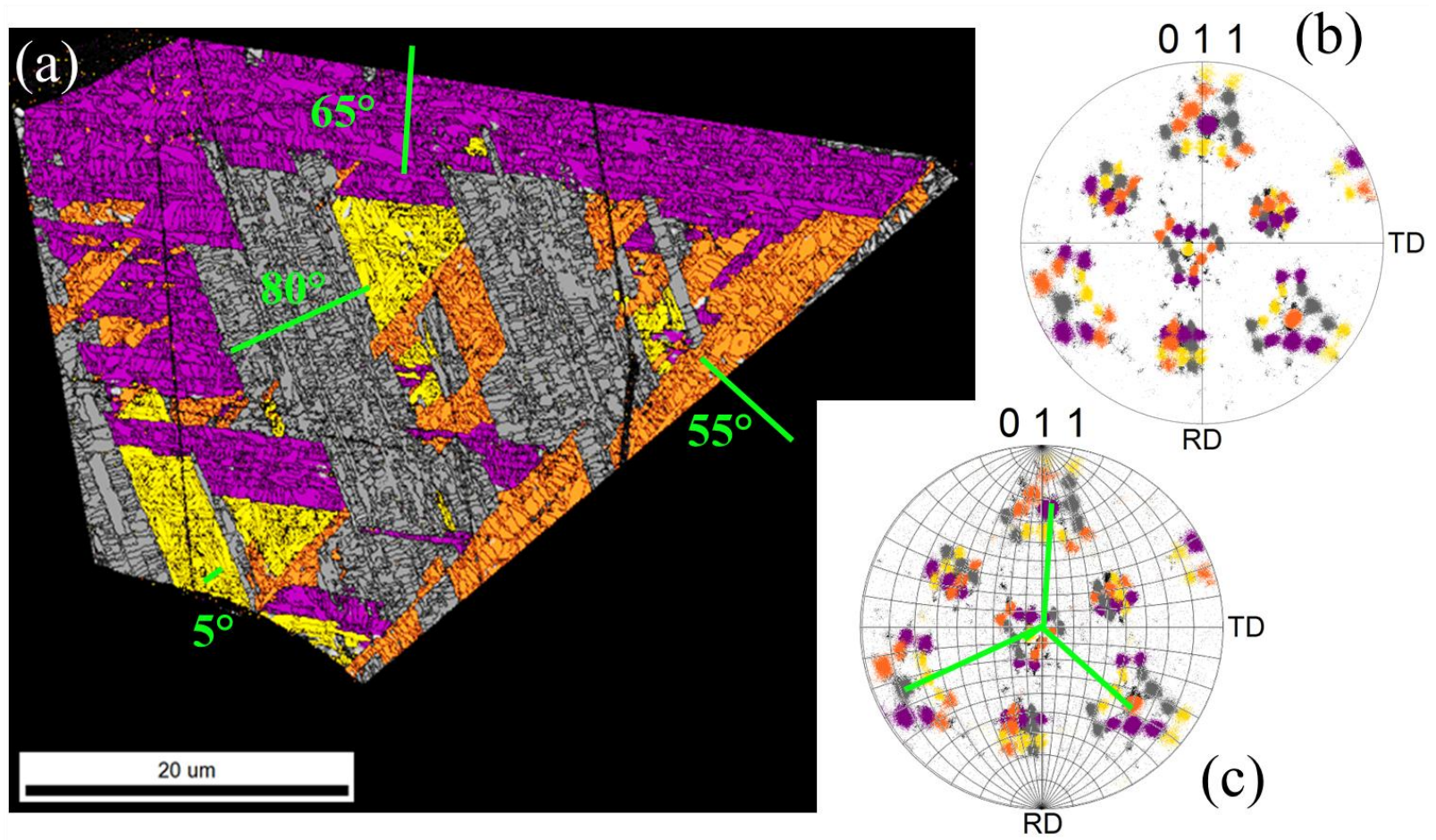

Fig. 6: (a) EBSD map showing the division of the prior austenite grain into the 4 crystallographically distinct packets, colored orange, yellow, purple and gray. (b) Laths in a packet share a common $\langle 011\rangle$ axis, which appears at the center of one of the four rosettes in the (011) pole figure. (c) The projections of the 4 packet axes onto the plane of the figure. The angles shown are the angles between the packet axes and the normal to the figure. 

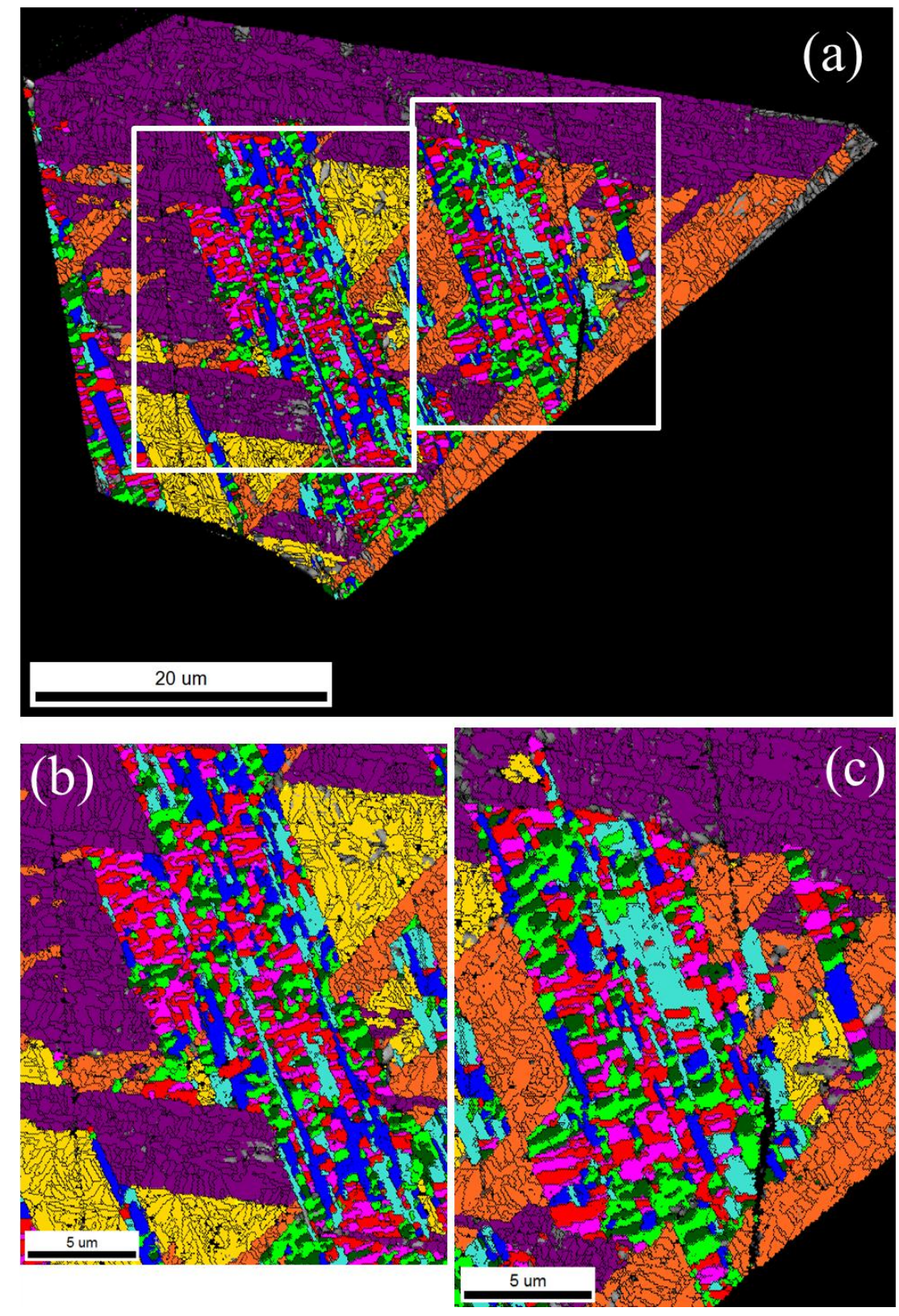

Fig. 7: (a) The prior austenite grain shown in Fig. 6, with the gray packet colored to show the KS variants of the individual laths, using the scheme defined in Fig. 4. $(b, c)$ Magnified views of the "gray" packet. The KS variants are organized roughly into parallel plates stacked perpendicular to the $[011]$ packet axis. Each plate contains all $6 \mathrm{KS}$ variants, organized in short segments of alternating twins. 


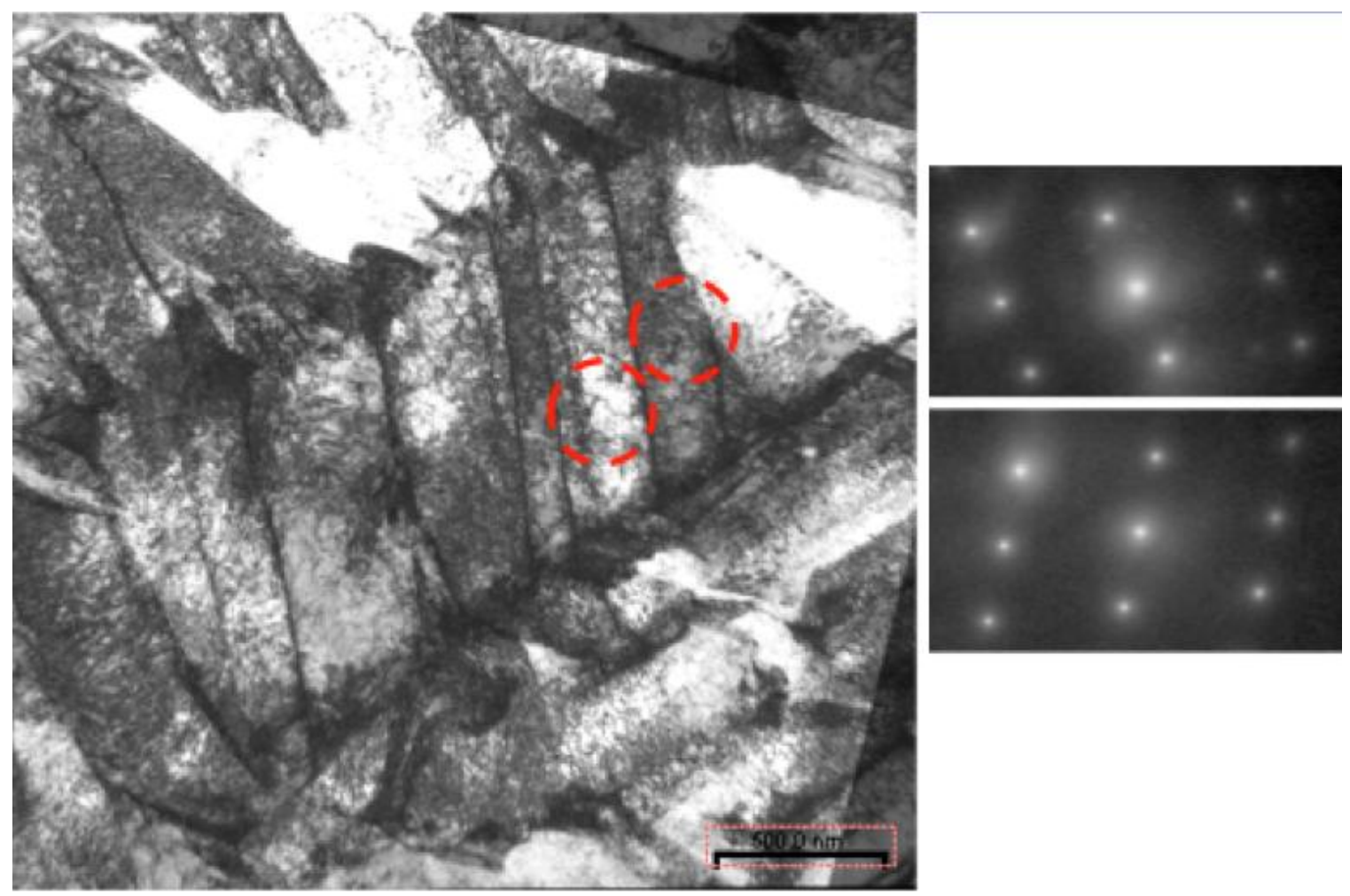

Fig. 8: TEM micrograph showing the cross-section of a plate in $12 \mathrm{Mn}$ steel. Diffraction patterns of the adjacent laths identified by red circles show that they are in twin orientation. 


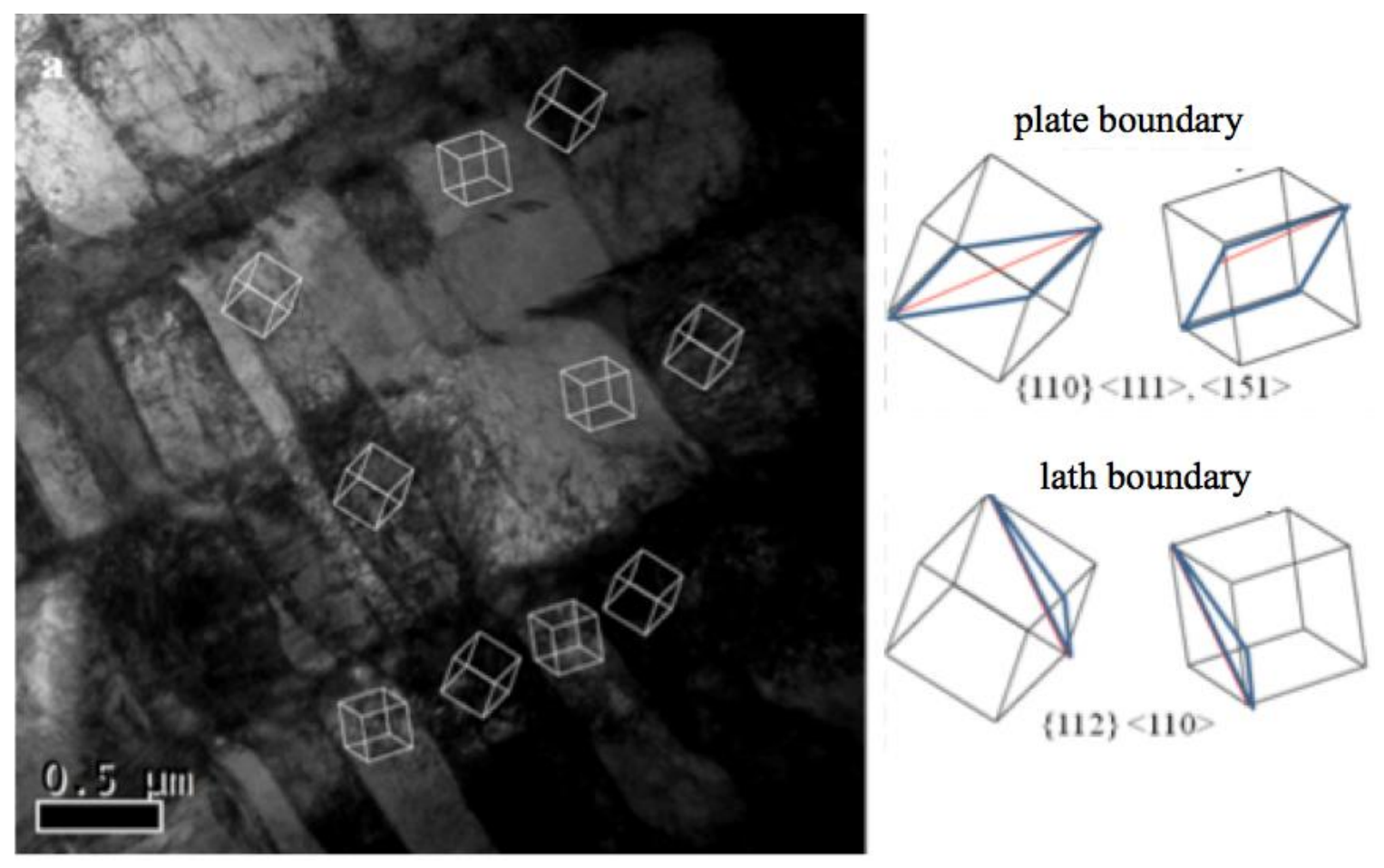

Fig. 9: TEM showing edge-on view of stacked plates. The plate boundary lies parallel to the common $(011)$ plane of the packet while the lath boundary is a $\{112\}$ twin plane. The red lines show the designated directions. 

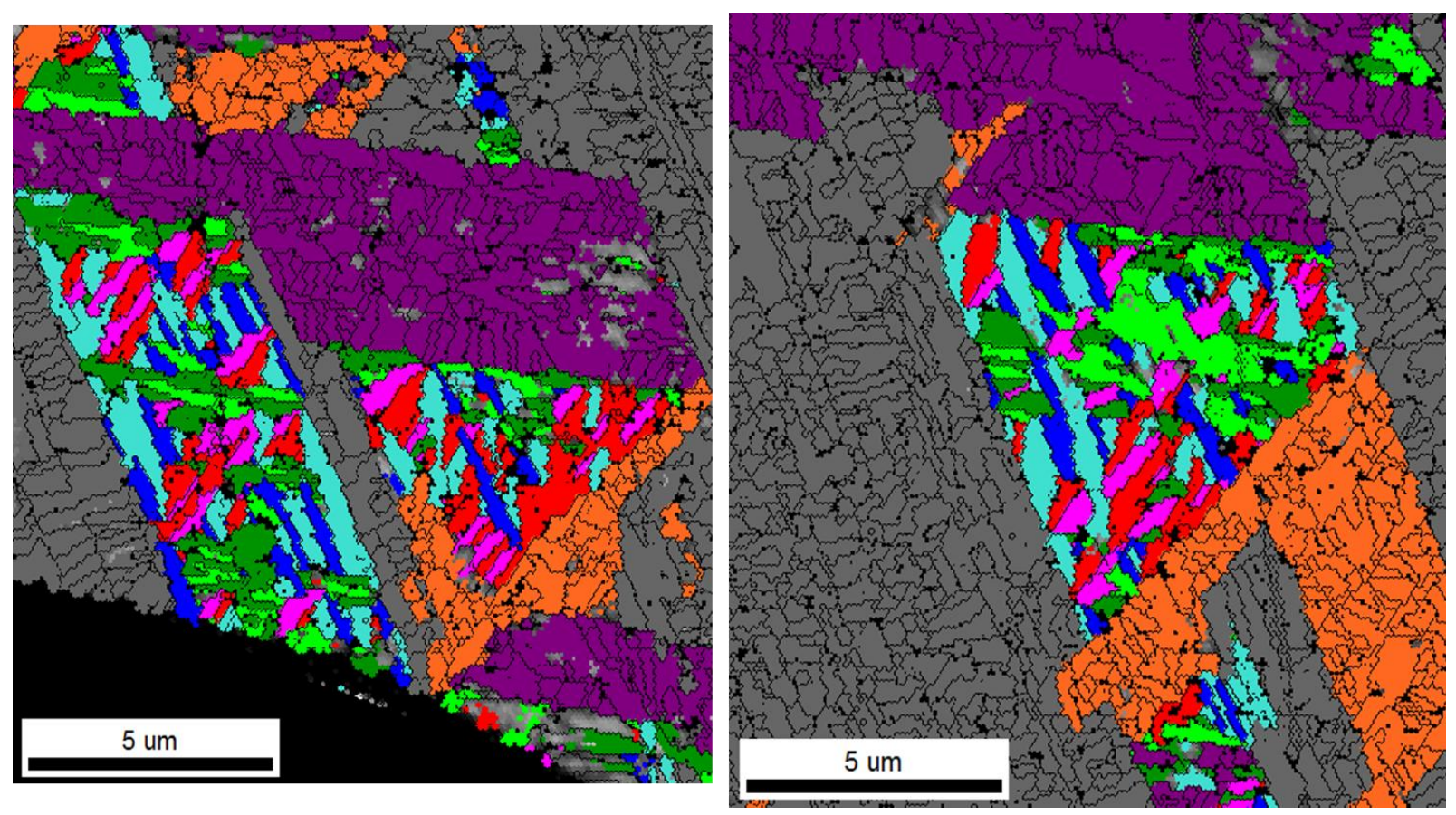

Fig. 10: KS variant distribution within the yellow packets of the grain in Fig. 6, in a view that looks down onto the plane of the martensite plates. The three sets of elongated twin pairs are oriented $120^{\circ}$ from one another, and interwoven in the plane of the plate, 

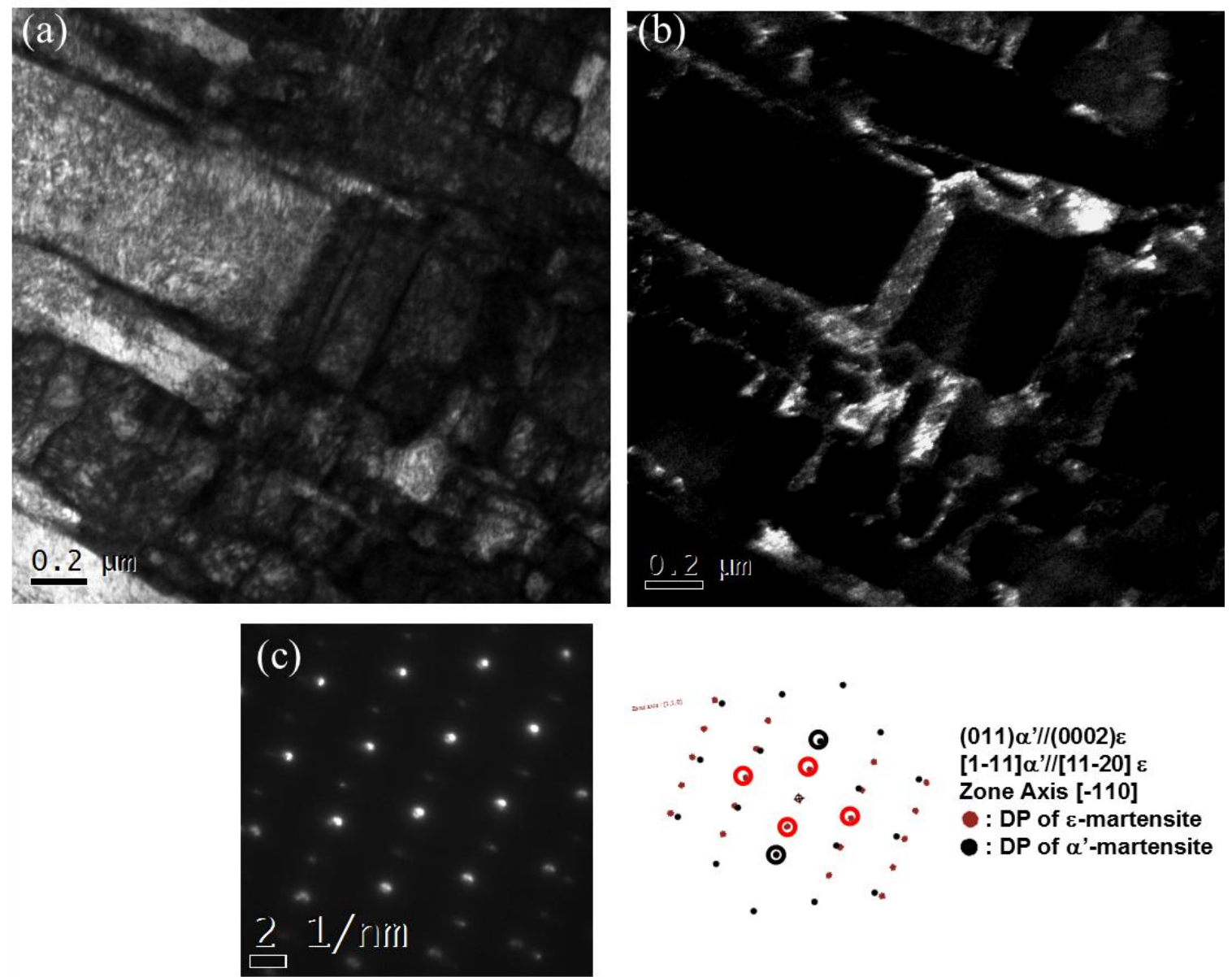

Fig. 11: (a) Bright-field TEM showing fine dislocated lath structure of asquenched $12 \mathrm{Mn}$ steel. (b) Dark-field micrograph highlighting the $\varepsilon$ phase in the microstructure. (c) Diffraction pattern showing $\alpha^{\prime}$ and $\varepsilon$ martensite peaks. 

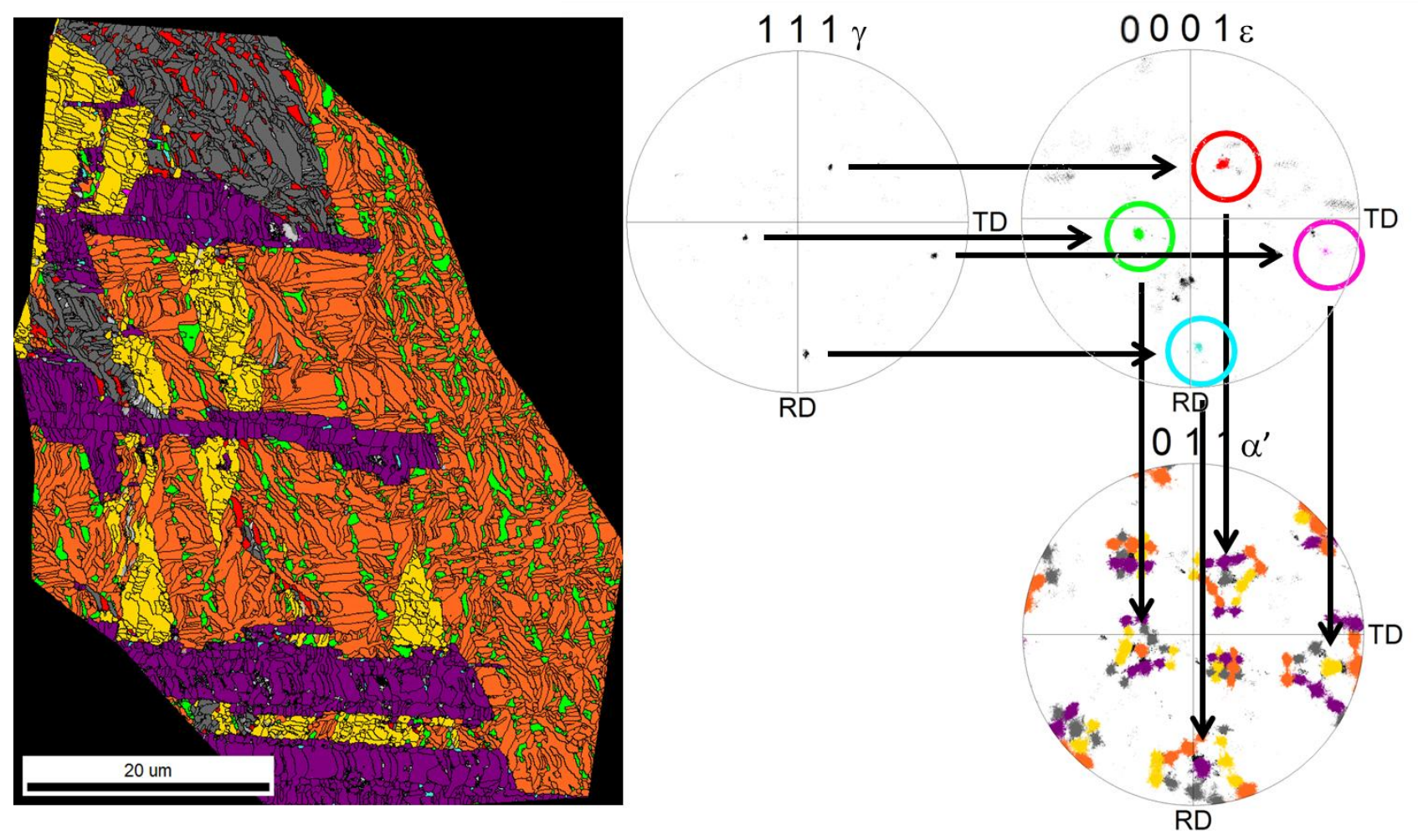

Fig. 12: Prior austenite grain in $12 \mathrm{Mn}$ steel with $\alpha^{\prime}$ packets and $\varepsilon$ variants colored green, red, magenta and blue. The pole figures at right show, respectively, the 4 (111) poles of the retained $\gamma$ phase, the 4 (0001) poles of the associated $\varepsilon$ phase, and the four (011) poles of the $\alpha$ ' packets. 

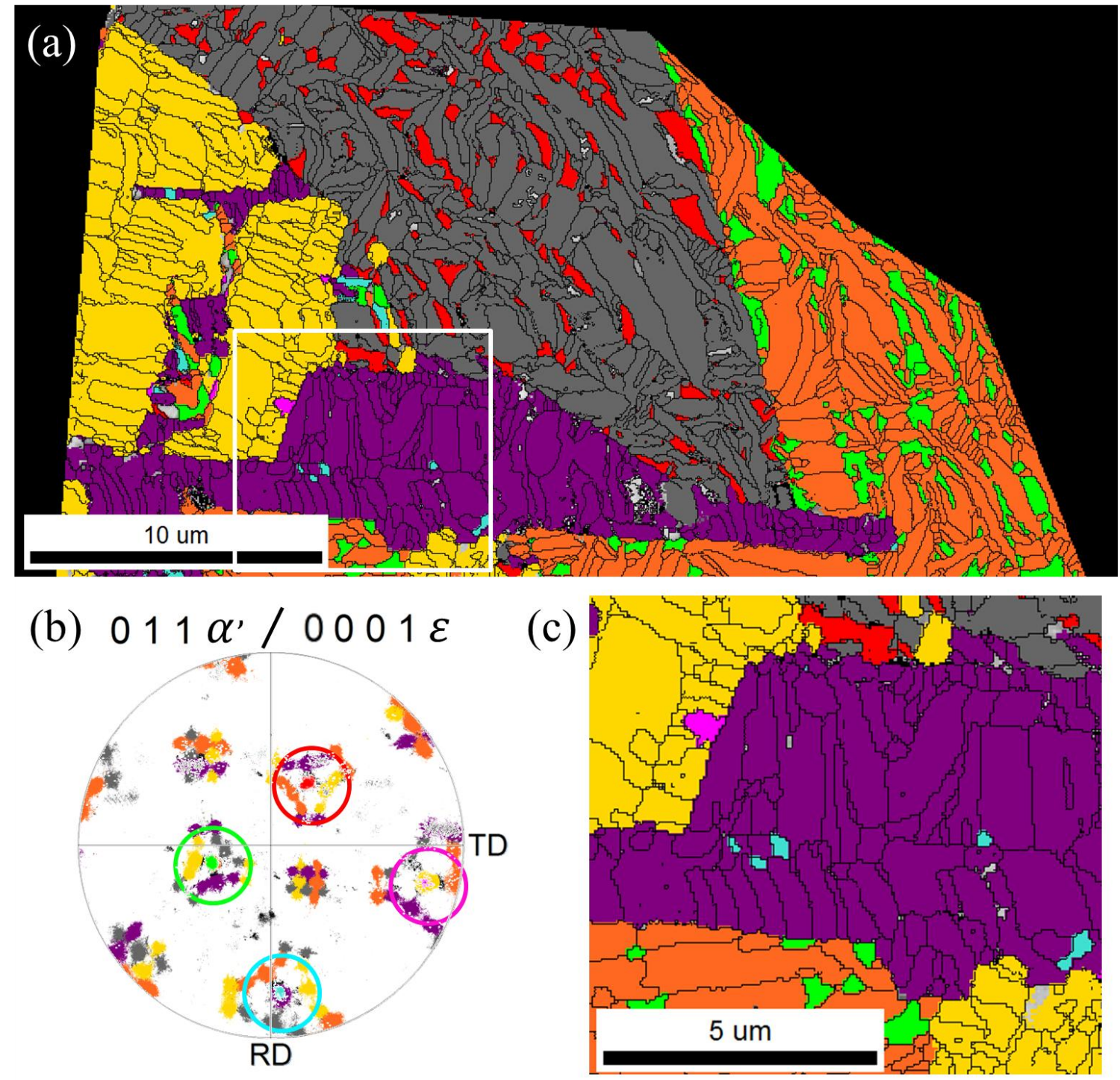

Fig. 13: (a) Magnified view of the grain shown in Fig. 12 with $\alpha$ ' packets and $\varepsilon$ variants colored. There is a unique association between the packet and the single $\varepsilon$ variant that appears within it. (b) The (0001) $\varepsilon$ pole figure is overlaid onto the (011) $\alpha^{\prime}$ pole figure and the orientation relations defined in eq. (2) are seen experimentally. (c) A high magnification view of the $\varepsilon$ variant and $\alpha^{\prime}$ packet relationship. 


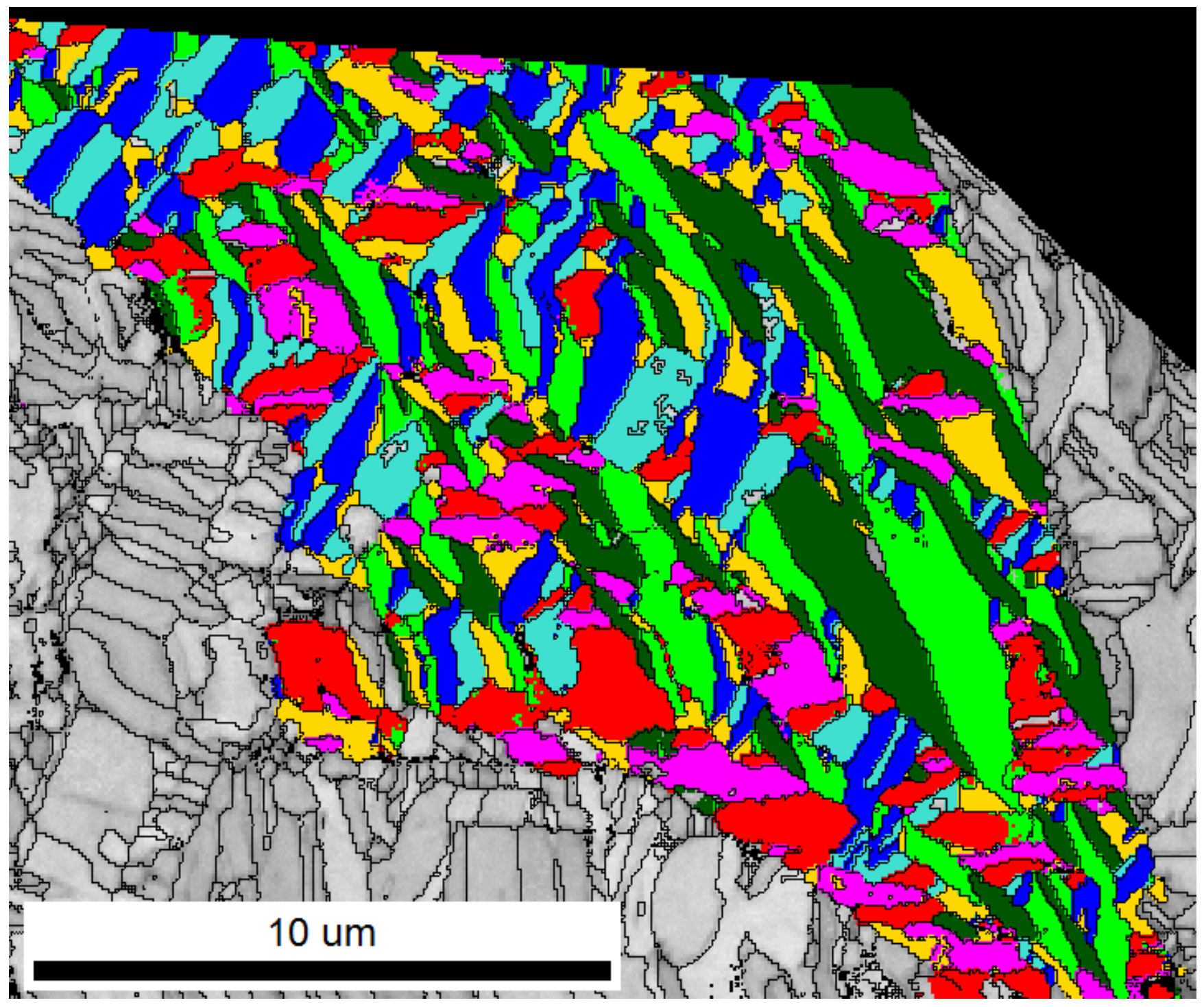

Fig. 14: Detail of the "gray" packet in Fig. 13 showing the distribution of the $6 \alpha$ ' variants colored as in Fig. 5, and the $\varepsilon$ phase, colored yellow. Twinned $\alpha$ ' variants are different shades of the same color. 


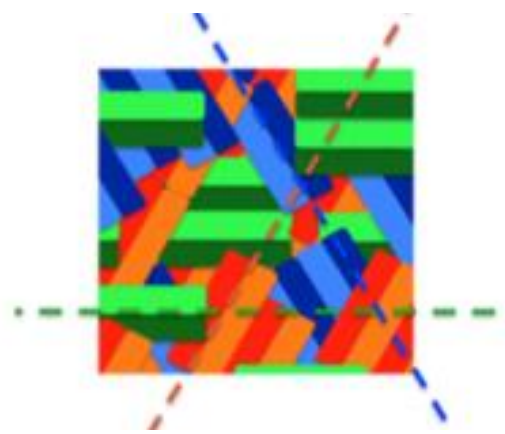

(a)

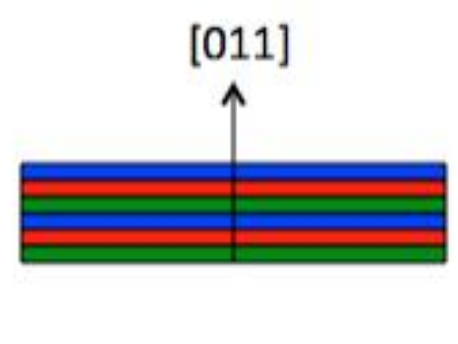

(b)

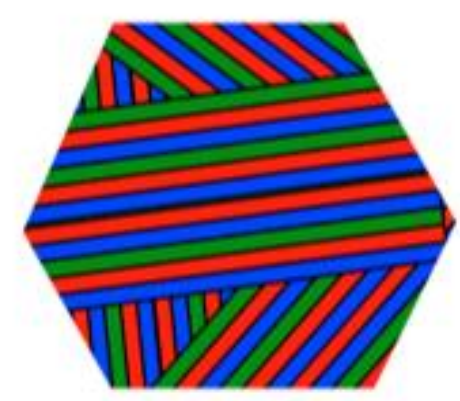

(c)

Fig. 15: The microstructure of $12 \mathrm{Mn}$ steel. (a) The building block is a 6-variant plate made up of interwoven twin pairs of KS variants. (b) The plates are stacked on their common $\{011\}_{a}$ plane to make a packet. (c) The four crystallographically distinct packets fill the prior austenite grain, producing a net strain that is a pure dilatation. 


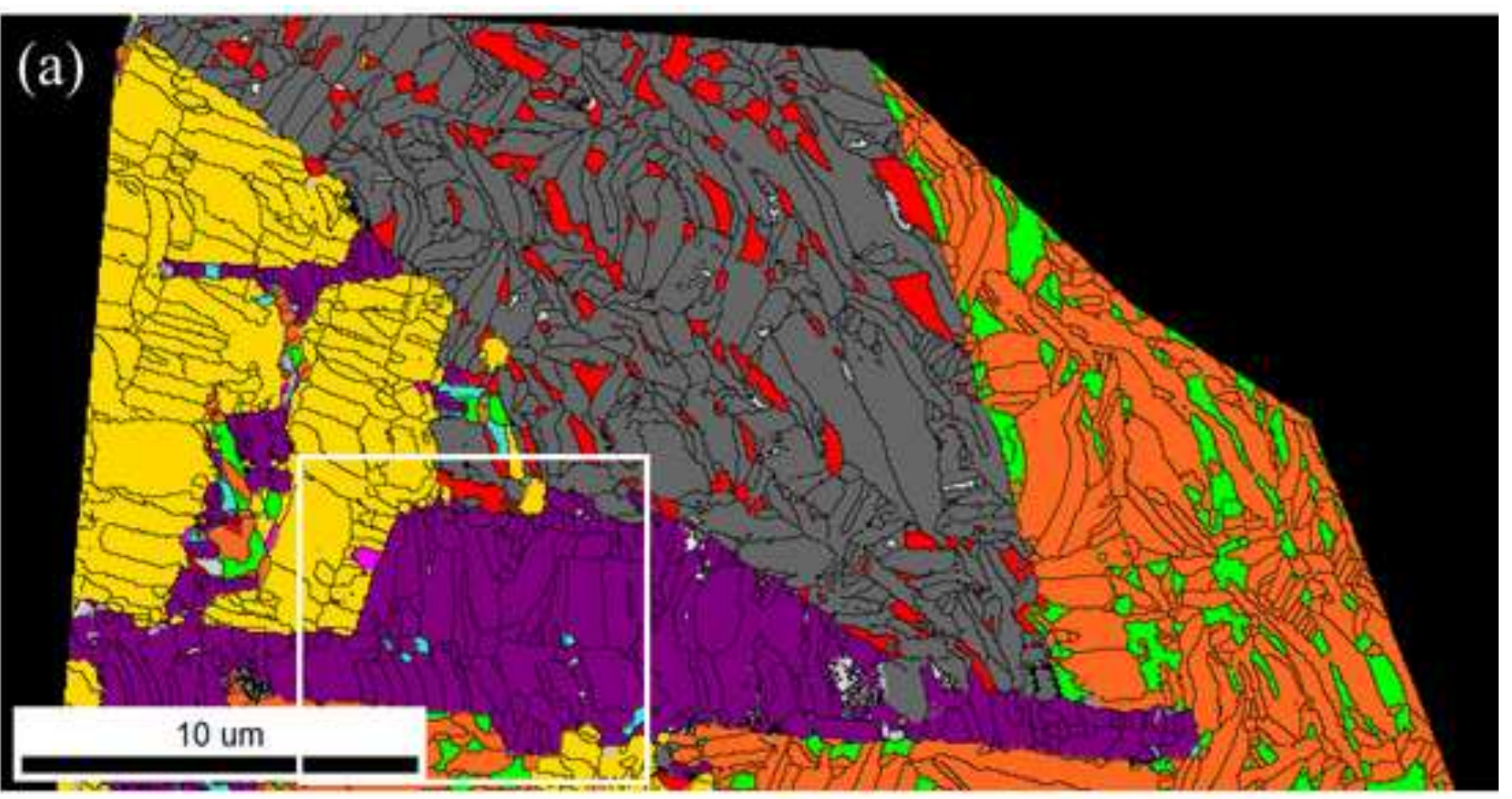

(b) $011 \alpha^{\prime} / 0001 \varepsilon$

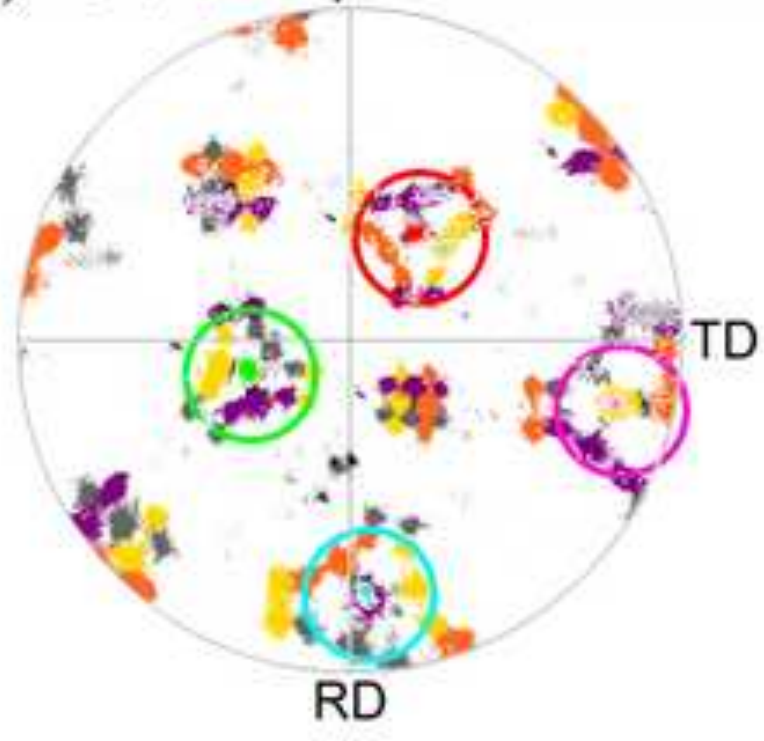

(c)

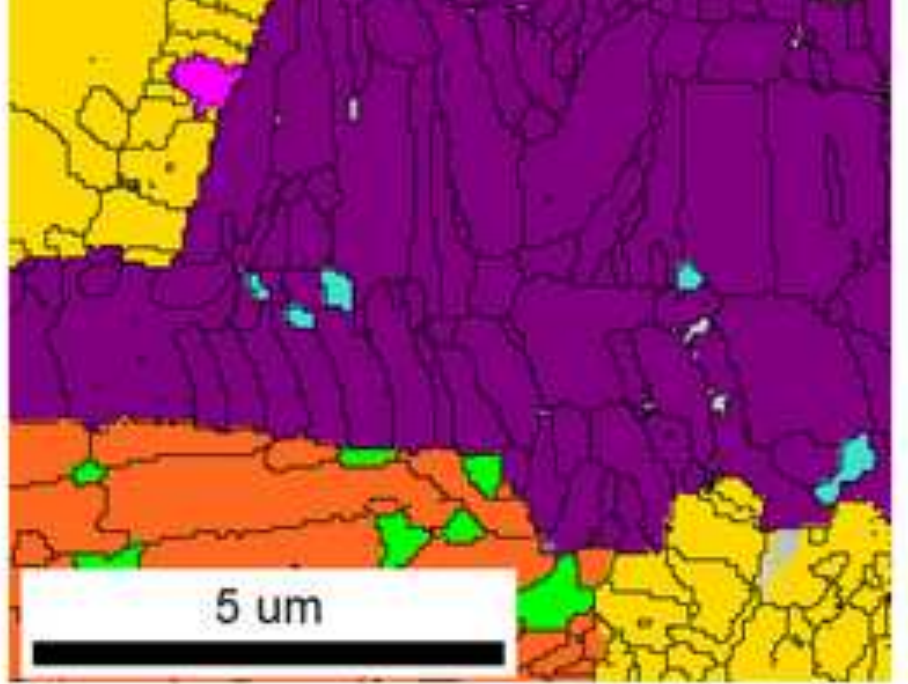

